\title{
Il trattato
}

di Anassimandro sulla terra

\section{LIVIO ROSSETTI / Perugia /}

1. Il sole che passa sotto la terra; 2 . La terra sospesa nello spazio e le ragioni della sua stabilità; 3. Apeiron ed infiniti mondi? 4. La terra a forma di cilindro o rocchio di colonna; 5. L'ideazione del proto-pinax e di una AU con cui ,misurare' perfino il cosmo; 6. Altre tessere, non così ben stabilite, del sapere cosmologico di Anassimandro; 7. Il cielo sopra di noi; 8. Dai grandi spazi ai grandi tempi: il passato remotissimo della terra. Anassimandro evoluzionista; 9 Il processo di essiccamento della terra; 10. La preistoria dell'ecosistema in cui noi ci troviamo a vivere. Asse spaziale e asse temporale; 11. „Mettersi dal punto di vista della terra” e affermare senza sapere; 12. Verso una conclusione: dove collocare la nozione di apeiron e il fr. 1? 13. Il libro di Anassimandro; 14. Infine: Anassimandro e Talete.

Sappiamo chi fu e che cosa ha insegnato Anassimandro? Sull'informazione di base, che è notoriamente disponibile dal 1903 - anche se dal 2011 possiamo contare sulla importante nuova edizione preparata dal Wöhrle -, grava un ben stabilita rinuncia a tentare di mettere ordine tra le varie tessere del mosaico, rinuncia che si combina con qualche 
specifica forma di ricorrente disattenzione. Per effetto di un simile orientamento esegetico ${ }^{1}$, si è affermata una immagine molto depauperata - e certamente molto poco ,caritatevole' - di Anassimandro. Viceversa un recente libro di Carlo Rovelli (2009, 2011) ha avuto l'impagabile merito di attirare l'attenzione su un nucleo dottrinale di pregio che è passato inosservato per un tempo straordinariamente lungo. Da questo nucleo dottrinale propongo di iniziare la presente nuova ricognizione degli insegnamenti del secondo maestro di Mileto.

\section{Il sole che passa sotto la terra}

Rovelli si concentra sulla disponibilità di Anassimandro a pensare che, di notte, il sole non possa far altro che continuare il suo viaggio sotto la terra, e che luna e stelle possano solo regolarsi allo stesso modo. L'idea è talmente elementare - e talmente familiare a tutti noi - che diventa difficile trattarla come una scoperta, tuttavia faremmo bene a ricordare che, secondo Anassimene, il sole al tramonto non prosegue il suo viaggio sotto la terra, ma fa un percorso di tipo orizzontale (verso nord, solo che noi non ce ne accorgiamo a causa delle montagne: $13 \mathrm{~A} 14 \mathrm{DK}=\mathrm{Ar} 4 \mathrm{~W}$.), dunque un percorso pur sempre semicircolare, ma che, ogni dodici ore circa, passa da un piano di rotazione approssimativamente verticale ad uno approssimativamente orizzontale. Di Anassimene ci viene infatti riferito che, di notte, «gli astri non passano sotto la terra (ou kineisthai hupo gèn ta astra legei) ma le girano attorno come un berretto» (13A7 DK = As 56 [7.6] W.; cf. $13 \mathrm{~A} 14=$ Ar 38 W.) o «come un macina da mulino» (13A12 DK = As 112 W. $)^{2}$. Barnes $(1979$, I 27) e altri propongono di intendere che Anassimene, poi Senofane ed Eraclito, si limitarono a passare da una terra matematicamente sospesa (per una ragione astratta) ad una terra fisicamente sospesa nell'aria. Ma se il sole non può passare di sotto e ,deve' cambiare il suo piano di rotazione ogni dodici ore circa (per Anassimene), oppure deve formarsi ex novo ogni mattina a est (per Senofane ed Eraclito³), ciò significa che, malgrado si parli

${ }^{1}$ Kahn (1960), Barnes (1979) e Kirk-Raven-Schofield (1983) costituiscono, a mio avviso, le principali occasioni mancate malgrado i molti meriti che a queste opere vengono giustamente riconosciuti. - In effetti mi trovo a difendere un'idea di Anassimandro molto diversa da quella che si è affermata nel corso dell'intero Novecento. Perciò non sorprende che questa affermazione risulti sorprendente, ma confido che le ragioni per affermarlo affioreranno piuttosto chiaramente dall'insieme della presenta indagine (v. in particolare le note 7 e 40 ).

2 Segnalo che, nell'ediz. Wöhrle, Th sta per Talete, Ar per Anassimandro e As per Anassimene.

3 Senofane poté scrivere (fr. $28 \mathrm{DK}$ ) che la terra ha un limite superiore ben visibile (laddove cede il posto all'aria) mentre, in basso, si estende all'infinito (cioè non ha un analogo limite inferiore). Poté inoltre sostenere, contro Anassimene, che la terra non è avvolta nell'aria e tantomeno sostenuta dall'aria (21A32 e $33.3 \mathrm{DK})$, e così pure che il sole è una mera concrezione gassosa che si riforma ogni giorno (21A32, 33.3, $40 \mathrm{DK})$ e che si dissolve ad ogni tramonto (per cui non c'è più bisogno di ipotizzare un percorso su due diversi piani di rotazione). Sull'effimero successo della cosmologia 'sbagliata' di Anassimene e Senofane è ora disponibile un mio studio, Rossetti (2013b) (ma v. anche Barnes 1979, vol. I: 26 s.). 
di mere masse d'aria, Anassimene e seguaci semplicemente negano che, sotto la terra, ci sia uno spazio vuoto o semivuoto in cui il sole possa completare il suo tragitto.

Se negano, allora qualcun altro aveva già affermato il contrario (che il sole può ben passare). Anassimene, intellettuale al quale cospicue benemerenze vanno comunque riconosciute, ha creduto di poter proporre una più rassicurante tesi alternativa, associata al rigetto dell'idea che la terra potesse essere 'sospesa nel vuoto' e, così facendo, si è assunto la responsabilità di gettare il discredito su alcune delle più ardite idee del suo diretto maestro e di ritornare, su un tema qualificante, alla communis opinio di greci e non greci. Aggiunge Rovelli: «il salto concettuale dalla Terra-piatta alla Terra-corpofinito che galleggia nello spazio» è stato «gigantesco e difficile. Lo prova il fatto che non ci sono arrivati i Cinesi in venti secoli di Istituto astronomico imperiale e non ci è arrivata nessun'altra civiltà» (2011: 52).

In effetti, unicamente Anassimandro ${ }^{4}$ osò rappresentarsi la terra come un corpo che è limitato non solo, di sopra' ma anche, di sotto', e gli astri come corpi che, nel loro moto circolare, passano tanto sopra quanto sotto la terra. Inoltre spetta a lui il merito di aver argomentato che la terra non cade (cioè: non cade quantunque abbia la possibilità teorica di precipitare nel vuoto, perché sotto c'è il vuoto), ma rimane ferma in posizione di equilibrio. Queste due tessere del sapere di Anassimandro sono positivamente documentate, mentre per la tessera individuata da Rovelli manca una analoga documentazione diretta. Ma perché mai Anassimandro avrà avvertito il bisogno di rappresentarsi il sotto della terra ed avrà condotto una profonda e creativa (quanto inedita) riflessione sulle ragioni per cui la terra è stabile pur essendo circondata da un immenso vuoto? Si richiede di aver prima raggiunto la conclusione che il moto del sole (e degli altri corpi celesti) ,deve' necessariamente proseguire in modo uniforme anche nelle ore notturne ed essere circolare.

Che sia stato questo il punto zero delle sue riflessioni sui rapporti spaziali è del tutto verosimile. Rispetto alla tesi del moto circolare degli astri, la convinzione che debba esistere un lato inferiore della terra è un corollario, mentre l'offerta di ragioni per sostenere che la terra non cade (e non corre alcun serio rischio di cadere) costituisce una tesi in grado di fornire un prezioso supporto all'intuizione primaria, ma complementare e nettamente distinta.

Qual è dunque l'intuizione primaria? dovrebbe essere questa: che nelle ore notturne il sole non interrompe e non modifica il suo corso. Intuizione elementare, verrebbe da dire, ma intuizione che cozza con altre intuizioni non meno elementari: perché mai la terra in quanto tale non cade mentre tutti i corpi (dall'acqua del mare alle persone) cadrebbero indefinitamente verso il basso se non ci fosse la terra a sostenerli? La spinta a presumere che ciò accade perché qualcos'altro la sostiene è quasi irresistibile! Inve-

\footnotetext{
${ }^{4}$ Faccio presente che c'è anche un'ipotesi alternativa, secondo cui, nella elaborazione di una così ardita opinione, Talete potrebbe aver anticipato il suo allievo. Se ne discuterà fra un momento (nel corso di questo stesso paragrafo).
} 
ce Anassimandro, come sappiamo, ha tenuto fermo il moto circolare dei corpi celesti e, di conseguenza, ha cercato e trovato una spiegazione alternativa del fatto che la terra non cade. E a quel punto ha potuto anche osare rappresentarsi il lato inferiore, antipodale della terra. Perveniamo, con ciò, a intuire quale poté essere il corso dei suoi pensieri.

Se dunque egli fu il primo - e per qualche tempo il solo, sempre che a lanciare l'idea non sia stato Talete (su ciò v. più avanti) - a sostenere che, di notte, gli astri continuano e completano il loro tragitto passando sotto la terra, ciò significa che l'Occidente ha appreso questo insegnamento epocale, del tutto qualificante per la nascita dell'astronomia greca e moderna, da Anassimandro malgrado il fuoco di sbarramento prontamente immesso in circolo da Anassimene (con ulteriori apporti dovuti a Senofane e altri). Così stando le cose, dobbiamo certamente chiederci chi ebbe il merito di riscoprire e rilanciare l'idea di cosmo propugnata senza successo da Anassimandro (deve essersi trattato di Parmenides). Ma a maggior ragione, e già solo per i motivi sopra richiamati, dobbiamo disporci a ravvisare nell'insegnamento di questo antico maestro un lascito di primaria importanza. Pertanto dobbiamo disporci anche a diffidare della riduzione dei suoi insegnamenti a mere doxai irrelate e giustapposte. Ed è interessante notare che cosa accade se, invece di svolgere considerazioni sul tipo di quelle appena offerte, cominciamo col dire che, secondo Anassimandro, la terra ha forma cilindrica, ha uno spessore pari a un terzo del suo diametro ed è in grado di librarsi nello spazio ${ }^{6}$ : riformulate in questo modo, le sue doxai non appaiono più interdipendenti e si perde di vista il pensiero che c'è dietro, fino al punto di lasciarsi sfuggire non questo o quel dettaglio, ma l'essenziale, il punto di partenza, la premessa decisiva.

Un cenno ora su Talete e su un dettaglio solitamente ignorato. Quando riferisce che Talete riuscì a stabilire che la grandezza apparente del sole è pari a 1/720, Diogene Laerzio non parla di 1/720 del cerchio, ma della «settecentoventesima parte del circolo solare» (tou heliakou kyklou ... heptakosioston kai eikoston meros: $11 \mathrm{A1} .24 \mathrm{DK}=$ Th $237.24 \mathrm{~W}$.); analogamente Apuleio, quando parla di questo stesso argomento (11A19 = Th $178 \mathrm{~W}$.), scrive che «potei verificare io stesso quante volte il sole divide, con la sua grandezza, la circonferenza che esso percorre» (comprobavi, quoties sol magnitudine sua circulum quem permeat metiatur). Ci viene dunque riferito che Talete avrebbe confrontato l'ampiezza angolare del sole con il percorso circolare (cioè diurno + notturno) del sole. Il fatto che una singolarissima scoperta di Talete venga posta in relazione al supposto circolo solare incoraggia pertanto a presumere che, prima di Anassimandro, possa essere stato Talete a pensare ad un sole che passa sotto la terra, tanto più che il dato è del tutto univoco e di conseguenza non è trascurabile. L'essenziale della benemerenza su cui si è soffermato Rovelli sembra insomma sul punto di essere trasferita da Anassimandro a Talete. Si deve però considerare che in Talete la notizia rimane priva di contesto e di sviluppi, mentre

\footnotetext{
${ }^{5}$ Nel caso di Parmenide si registra l'analogo uso di non riconoscere l'epocale importanza della sua 'scoperta' concernente la sfericità della terra.

${ }^{6}$ Di questo genere è il resoconto proposto in KRS (1983: $\left.133 \mathrm{~s}\right)$.
} 
ben diverso è il caso di Anassimandro. Di conseguenza, sul possibile contributo di Talete è doveroso mantenere un sostanzioso punto di dubbio.

Come si vede, vengono maturando le condizioni per un complessivo ripensamento degli insegnamenti di questo antico maestro, ed ha senso chiedersi non da quale idea di Anassimandro partiamo, ma verso quale idea stiamo muovendo. Infatti c'è tutto un Anassimandro sommerso di cui bisognerà pur cominciare a tenere il debito conto, visto che finora la comunità scientifica ha accreditato un'immagine molto impoverita del personaggio e delle sue straordinarie acquisizioni.

\section{La terra sospesa nello spazio e le ragioni della sua stabilità}

Delle due idee correlate al percorso circolare del sole, il tema dei limiti inferiori della terra si lega ad altre importanti escogitazioni e in modo particolare ad un giro di pensieri che non è in alcun modo riconducibile alle congetture sul percorso notturno che fa il sole. Il suo esame viene perciò rinviato (al $\$ 4)$.

Nel momento in cui ha raggiunto la conclusione che la terra dovrebbe avere anche un limite inferiore, per cui un grande vuoto dovrebbe esserci non solo sopra, ma anche sotto e tutto attorno ad essa, Anassimandro deve essersi chiesto se è concepibile che la terra, corpo fisico di grandi dimensioni circondato da astri lontani, se ne stia in condizione di quiete all'interno di un cielo (o spazio, o vuoto, o aria, o apeiron) immenso, senza bisogno di alcun sostegno per non cadere. Perché non cade la terra nel suo complesso, così come non cadono sole, luna e stelle? Possiamo farci almeno un'idea del sistema di relazioni che permette a tutti questi corpi di coesistere in modo ordinato e, per così dire, senza scosse? Pensare di potersi misurare con domande di così immensa portata e di individuare risposte convincenti fu, presumo, un atto di suprema ambizione intellettuale ${ }^{7}-$ cosa $^{-}$ che, d'altronde, è del tutto congrua per un allievo di Talete.

In materia, le fonti ci offrono un aiuto più che considerevole. Il testimone più antico è Platone. Questi nel Fedone (108e-109a) scrive che, secondo Socrate (non secondo Anassimandro!), la terra è peripherēs, circolare (cioè sferica), se ne sta ferma nel mezzo del cielo e, per non cadere (pros to mē pesein), non ha bisogno nemmeno dell'aria ${ }^{8}$ perché, allo scopo, sono sufficienti la homoiotēs (,somiglianza', cioè uniformità? equilibrio? indifferenza? equidistanza? omogeneità? uguaglianza?) del cielo con se stesso in ogni sua parte e l'equilibrio (isorrhopia) derivante dalla sua forma sferica. Il Socrate platonico conclude

7 È noto che nel 1958 provvide Karl Popper ad attirare l'attenzione della comunità scientifica su questo estremo ardimento di Anassimandro. - Non posso non osservare, con l'occasione, che congetture così iperbolicamente ardite quali quella sul sole che passa sotto la terra e questa sulla terra che non cade (ma sappiamo bene che ve ne sono moltissime altre non meno eccelse) avranno verosimilmente appassionato l'autore, occupato e appagato la sua mente, in misura incomparabilmente superiore alle riflessioni sull'apeiron di cui si è ripetutamente interessato Aristotele.

${ }^{8}$ Si noti: questa fu la tesi di Anassimene e,con modulazioni diverse, Senofane. 
il suo ragionamento affermando che un oggetto così ben equilibrato non può inclinarsi da nessun lato. In altre parole, può solo rimanere come e dove è. Poiché si parla di terra sferica, il Socrate di questo passo è sicuramente espressione di una cultura astronomica più ,avanzata' di quella di Anassimandro, però manifestamente presuppone la domanda che poté prendere forma solo grazie all’insegnamento di Anassimandro („Perché non cade?") ed è verosimile che la rifletta da vicino.

Aristotele è più esplicito. Egli ha occasione di scrivere che, secondo alcuni (alcuni ,filosofi') come, tra i più antichi, Anassimandro, la terra sta ferma dia tēn homoiotèta, per via della sua ,somiglianza'. «Né verso l'alto né verso il basso o di lato - prosegue Aristotele - ha attitudine a muoversi (pheresthai prosēkei) ciò che è collocato al centro e sta allo stesso modo in rapporto agli estremi. D'altronde non potrebbe nemmeno muoversi simultaneamente in direzioni opposte, sicché è necessario che <la terra > stia ferma. Tutto questo è molto ben detto, ma non veritiero» (De caelo II 13, 295b11-16 = 12 A26 $\mathrm{DK}=\mathrm{Ar} 6$ W.). Come si vede, Aristotele è inequivocabile nell'asserire che Anassimandro fu il primo o uno dei primi (ma non il solo) ad argomentare che, se la terra non cade, è perché non ha motivo di cadere né dispone di una direzione privilegiata verso cui eventualmente cadere, e questo è precisamente il ragionamento fatto dal Socrate platonico. Anche la precisazione finale, «Tutto questo è molto ben detto, ma non veritiero (kompsōs men, ouk alèthōs de)», merita attenzione. Per il fatto di affermare che il ragionamento è ben costruito e la dimostrazione è ,bella' (cioè attraente, intuitiva, in grado di convincere) anche se non vera, Aristotele mostra di fare riferimento a un testo, a un ragionamento di qualche antico autore, e per l'appunto egli ha occasione di precisare che Anassimandro si distinse tra gli antichi (dunque escludendo Platone) nel sostenere questa tesi. In teoria, la tesi di Anassimandro avrebbe potuto essere stata ripresa da altri presocratici, ma da ciò non consegue un serio dubbio sull'eventualità che Aristotele qui faccia riferimento proprio agli argomenti con cui Anassimandro difese la sua tesi e fors'anche alle parole con cui la enunciò.

Quanto poi agli altri intellettuali che, secondo Aristotele, condivisero questo particolarissimo ragionamento, è disponibile una segnalazione dossografica (28A44 DK = $\mathrm{T} 75$ Coxon) secondo cui Parmenide e Democrito teorizzarono in modo analogo l'equilibrio dovuto alla uguale distanza da tutti i lati. Ora, che Parmenide abbia avuto il merito di riscoprire e rilanciare l'astronomia di Anassimandro è tesi raramente affermata, ma facile da argomentare e in grado di resistere a molte obiezioni. Pertanto è ragionevole pensare che Aristotele intendesse alludere proprio a Parmenide (ed eventualmente a Democrito), oltre che al maestro di Mileto.

Congruente è anche l'informazione fornita da fonti posteriori. Intorno al 200 d.C. Ippolito ha occasione di riferire che, per Anassimandro, la terra è «sollevata senza essere sostenuta da alcunché, ferma per via della sua uguale distanza da tutto» (Refutatio I 6.3 = 12A11 DK = Ar 75 W.). Intorno al 540 Simplicio specifica due volte che per Anassimandro e Platone la terra sta ferma dia tēn isorropian kai homoiotēta, «grazie all'equilibrio e alla somiglianza» (Commento al De caelo di Aristotele VII 520-21 e $532=$ Ar 187 e 189 W.). Intorno al 1250-1255 è poi Alberto Magno a ritornare con competenza 
sull'argomento nel suo De caelo et mundo. Al tema egli dedica una dettagliata e ben argomentata esposizione ${ }^{9}$ in cui da un lato conferma ciò che sappiamo da altre fonti (scrive ad es.: dixit enim iste, quod terra in medio fixa est quieta, eo quod est involuta in centro propter aequalitatem distantiae ab horizonte per circuitum. opinabatur enim, quod omne quod in medio stat <et> aequaliter distat a circumferentia horizontis, non magis habet moveri ad unam partem quam ad aliam et ideo ad nullam partem declinat), mentre dall'altro insiste nel sostenere quod ipsa distantiae aequalitas causa sit quietis terrae et non natura aliqua, quae sit in terra (II 4.6 = Ar 266 W. $)^{10}$.

Possiamo dunque dire di sapere per certo che Anassimandro - e nessun altro nel corso del VI secolo a.C. - pervenne ad argomentare che la stabilità della terra non è dovuta ad una causa fisica esterna (ad es. nel senso che si appoggia a, ed è sostenuta da, una macro-entità diversa dalla terra) ma alla sua condizione di equilibrio, condizione descrivibile anche in termini degni dell'asino di Buridano: „non avrebbe saputo da che parte cadere". Fermo rimane che il punto di partenza sia sempre il sole, che si presume sia in grado di passare tanto sopra quanto sotto la terra. Se questo è ciò che accade, allora non solo non c'è nessun piedistallo cosmico sul quale la terra sia appoggiata, ma è comprensibile che non ce ne sia bisogno. Infatti si constata che la terra è inserita in un sistema di corpi celesti in movimento in cui gli spostamenti sono ordinati, regolari, prevedibili e sostanzialmente privi di imprevisti. Quindi si deve solo costruire una spiegazione a partire da questa constatazione.

I passaggi logici appena delineati sono decisivi, credo, per poter poi parlare di una situazione in equilibrio in cui la terra sta ferma, per così dire, senza sforzo. E se la congettura è fondata (come oso credere), allora noi siamo penetrati nel mondo mentale di Anassimandro, abbiamo capito come egli ha condotto la sua riflessione e la sua ricerca.

Perché dunque il sistema è stabile? Perché è in equilibrio, perché è homoios in tutte le direzioni, perché se ci poniamo dal punto di vista del cosmo (come dobbiamo, e si noti che certamente nessuno al mondo prima di Anassimandro era stato capace di porsi dal punto di vista del cosmo) non notiamo asimmetrie o altre disuguaglianze. Notiamo, al contrario, che non si ha notizia di nessun fattore di disturbo che possa ragionevolmente incidere sugli equilibri del sistema ,terra + corpi celesti' immerso in uno spazio indefinitamente grande e, si può presumere, vuoto se non si considera l'involucro aeriforme della terra. Quindi è normale e logico che il sistema continui a rimanere stabile, mentre l'ipotesi di una terra che cade (ma in che direzione?) implicherebbe l'intervento di una forza così grande da scompaginare tutto il sistema e dar luogo ad un cataclisma di proporzioni (diremmo noi) cosmiche. Se dunque non ci sono nemmeno remote avvisaglie di tale cataclisma, ciò vuol dire che il sistema è in equilibrio. Da qui il più che probabile - e tutt'altro che ingiustificato - passaggio dalla constatazione alla legittimazione. Da qui anche le

\footnotetext{
9 Segnalata per la prima volta, che io sappia, dal Wöhrle.

10 Platone è stato dunque il solo a svolgere le idee di Anassimandro senza dichiarare la sua fonte.
} 
prime avvisaglie di quel ciò che per noi sono il principio di inerzia e il principio di ragion sufficiente.

Di riflesso, la caduta dei gravi dall'alto in basso che noi sperimentiamo continuamente viene declassato: non viene più considerato una costante universale (o caratteristica peculiare della terra nel suo complesso) ma un fenomeno ,limitato', che trova applicazione soltanto sulla superficie della terra (anzi, in modo diverso su ciascuna delle sue due superfici piane). In altre parole, ciò che vale per i micro-fenomeni superficiali (la caduta dei gravi) non può valere per la terra considerata come un tutto unitario (un macrocorpo), perché in tal caso la situazione sarebbe squilibrata e profondamente diversa da quella che osserviamo ogni giorno ${ }^{11}$.

Che Anassimandro possa aver fatto e insegnato a fare simili ragionamenti è certamente sbalorditivo, ma abbiamo argomenti per non pensarlo? Non credo che ve ne siano: la tesi della stabilità da equilibrio (quasi una tautologia) è inequivocabilmente attestata. Dobbiamo perciò adattarci (sic!) all'idea che Anassimandro abbia ideato una spiegazione che fuoriusciva completamente dagli orizzonti intellettuali del suo tempo, e tutto questo per rendere comprensibile il supposto passaggio notturno del sole sotto la terra. In secondo luogo, dobbiamo adattarci (sic!) all'idea che egli abbia non semplicemente intuito, ma costruito un ragionamento che, riguardo alla stabilità del sistema, terra + corpi celesti', seguiva un percorso mai tentato prima da alcun essere umano. In terzo luogo abbiamo il privilegio di comprendere bene questo suo non semplice e non elementare percorso riflessivo e argomentativo. Ma provare ad esplicitarlo è possibile. Questa è la mia proposta di ricostruzione:

a. di notte il sole non può non completare il suo percorso passando sotto la terra;

b. quello dev'essere un percorso circolare;

c. anche nel caso di luna e stelle dovrebbe accadere qualcosa di analogo;

d. la terra deve dunque avere un limite inferiore ed è verosimile che, circondata come è dai corpi celesti e da nient'altro se non un grande vuoto (o spazio, o apeiron), se ne stia ferma ,in mezzo' a loro, in posizione centrale;

e. e ciò in virtù della condizione di equilibrio in cui si trova l'insieme di terra e corpi celesti;

f. di riflesso, il cadere degli oggetti materiali che noi quotidianamente osserviamo è una caratteristica di questo pezzo di mondo (una peculiarità di questo lato approssimativamente piano?) ma non della terra in quanto tale.

11 È quanto meno affascinante considerare che la spiegazione proposta si sarebbe potuta esplicitare sotto forma di contrapposizione tra la terra intesa come un tutto unitario di grandissime proporzioni, collocato al centro dell'universo, e la pietra che, rispetto alla terra, è piccolissima e quindi cade su di essa in virtù della sua estrema piccolezza, cioè perché 'dipende' da ciò che è infinitamente più grande di lei: ragionamento che già fa pensare a... Newton! 


\section{Apeiron ed infiniti mondi?}

Prima di procedere oltre sembra necessario affrontare due questioni collaterali. La prima concerne l'apeiron. Che io sappia, una relazione tra i pochi nuclei dottrinali fin qui esaminati e la teoria dell'apeiron non viene istituita da alcuna fonte. La possibilità di dare un nome allo spazio verosimilmente vuoto che circonda il sistema ,terra + astri' permetterebbe di dare un nome allo spazio che si presume possa estendersi indefinitamente oltre il sistema, ma si tratta di una eventualità totalmente congetturale. Pertanto, fatte salve le considerazioni svolte nel successivo $\$ 12$, propongo di non tenere conto di questa mera (anche se attraente) possibilità.

Si deve anche considerare l'eventualità che Anassimandro abbia teorizzato una infinità di mondi, che si susseguono o che coesistono. L'argomento è stato esaminato con molta cura dal Kahn, che ebbe anche cura di riunire le evidenze testuali pertinenti (1960: 46-53) e si osserva che l'autorità di Simplicio (Ar 178, 182, 192 W.) e delle fonti dossografiche si scontra con le perplessità che sono state passate autorevolmente in rassegna in KRS (1983: 122-126). Le esitazioni di Kahn e KRS appaiono pertanto ampiamente condivisibili. Del resto, tra la supposta infinità dei mondi e le altre tessere della cosmologia di Anassimandro non si intravede nessun raccordo plausibile. Pertanto sarebbe incauto rischiare di perdersi di nuovo in altre nebbie proprio mentre ci si adopera a dissolvere la fitta nebbia che ha sempre circondato la figura di Anassimandro e i suoi insegnamenti. Ne deduco che è desiderabile porre ogni cura nel mantenere distinte le informazioni non aleatorie (ce ne sono molte) e le questioni più difficili da decidere. Osservo inoltre, con l'occasione, che il desiderio di chiarire ed esplicitare concetti come archē e apeiron è tipico dei posteri che notano oscillazioni nel modo di impiegarli, mentre difficilmente costituisce una preoccupazione per chi ne inaugura l'uso. Ne deduco che faremo bene a non chiedere ad Anassimandro ciò che egli è verosimilmente impreparato a precisare, e a non costruire interpretazioni a partire da nozioni necessariamente mal definibili come quelle appena richiamate.

Riassumendo, dubito (ma non escludo) che la terre e gli astri siano stati concepiti come ,immersi' nell'apeiron, e dubito (ma non escludo) che possa essere stata lanciata anche l'idea dell' infinità dei mondi. Più semplicemente, nel tentativo di rappresentarsi il modo in cui Anassimandro ha elaborato la sua complessa idea delle relazioni spaziali, propongo di privilegiare le informazioni che, per nostra fortuna, non hanno basi così imprecise. 


\section{La terra a forma di cilindro o rocchio di colonna}

Passaggio successivo: la forma della terra. Sappiamo che Anassimandro (ancora una volta: solo lui) ha ipotizzato la forma cilindrica della terra, con due piani approssimativamente orizzontali e paralleli - dunque anche con gli antipodi ${ }^{12}$ - e una distanza tra i due piani pari a circa un terzo del diametro. Ippolito ed Aezio hanno occasione di riferire, fra l'altro, che secondo Anassimandro la terra somiglia ad un < rocchio di $>$ colonna di pietra (kioni lithōi: $12 \mathrm{~A} 11.3 \mathrm{DK}=\mathrm{Ar} 75 \mathrm{~W}$. e 12A25 DK = Ar 65 W.); inoltre Ippolito precisa, subito dopo, che uno dei due lati (cioè una delle due superfici approssimativamente pianeggianti) della terra è quello da noi occupato mentre l'altro è quello che sta dall'altra parte (antitheton). A sua volta Eusebio di Cesarea, che cita lo pseudo-Plutarco, precisa che, per Anassimandro, la terra è di forma cilindrica (kylindroeide $\overline{\text { ) }}$, con una profondità (bathos) del cilindro terrestre pari a un terzo della sua ampiezza (platos: 12A10 DK = Ar 101 W.). L'informazione è scarna, ma il riscontro c'è e, ancora una volta, l'informazione viene riferita al solo Anassimandro, per giunta da parte di intellettuali non particolarmente versati in astronomia, né animati da uno specifico interesse per la forma della terra, la possibile esistenza di antipodi etc. Di nuovo notizie inequivocabili, dunque, ma strabilianti, e anche piuttosto misteriose. Che senso poté mai avere, per Anassimandro, questo suo azzardarsi a dare addirittura delle misure?

Perché mai la terra sia stata concepita come costituita di due lati piani è comunque facile da capire: se il mondo a noi parzialmente noto è piano (o in prevalenza pianeggiante, o con alture comparativamente modeste), e se l'estensione della terra non è infinita, se d'altronde si presume che la terra a noi parzialmente nota abbia un profilo circolare e un apprezzabile spessore, perché non ipotizzare che il bordo esterno (l'argine esterno del fiume Oceano) dia poi luogo, come già Omero lasciava immaginare (Il. XVIII 608), a una sorta di invalicabile burrone e che, nell'altro lato, prenda forma un'altra area anch'essa approssimativamente pianeggiante? Non è pertanto azzardato presumere che Anassimandro si sia realmente spinto così lontano in congetture che furono parte integrante dei suoi insegnamenti (e del suo libro).

La circostanza obbliga però a pensare che il geniale sophos di Mileto sia stato capace di riflessioni straordinariamente disciplinate malgrado il loro estremo ardimento, tanto più che i dati disponibili si direbbero in grado di indirizzare le nostre congetture con esemplare disciplina intellettuale: se Omero ha ragione nell'insegnarci che la sua forma è circolare e che il bordo esterno del fiume Oceano non è oltrepassabile in quanto poi

${ }_{12}$ Si noti che, nel caso di una terra cilindrica, l'esistenza di una seconda superficie grosso modo pianeggiante implica (non può non implicare) che lì i corpi cadano nella direzione inversa a quella che si osserva nel mondo in cui ci troviamo a vivere noi e che l'acqua, in particolare, si depositi senza eccezione negli avvallamenti 'cadendo' nella direzione opposta. Il fatto di non sapere nulla intorno a questo specifico corollario (la direzione in cui i corpi cadono nell'altro lato pianeggiante) non prova che di esso non si facesse parola nel libro di Anassimandro. Aggiungo che un indizio a favore viene dalla storia evolutiva della terra. Infatti se Anassimandro poté congetturare un processo di formazione dei mari da un lato (cf. $\$ 10$ ), non è fuor di luogo presumere che egli abbia ipotizzato un processo analogo anche nell'altro lato. 
la terra strapiomba, se la terra ha un limite inferiore ed è stabile al centro del mondo, allora può ben avere una forma cilindrica. E se la superficie piana che agli elleni dell'epoca era solo parzialmente nota è così immensamente estesa, e se tale verosimilmente sarà anche l'altra, allora forse possiamo presumere che lo spessore sia un po' più contenuto.

Non si vuol dire, con ciò, che le considerazioni effettivamente svolte da Anassimandro (che non ci sono pervenute) coincidano con quelle ora abbozzate, ma è difficile immaginare che la distanza tra le une e le altre sia molto grande. La sostanza delle sue valutazioni è infatti del tipo indicato. Di conseguenza, dobbiamo adattarci ad ipotizzare che il secondo sophos di Mileto sia stato capace di elaborare congetture disciplinatissime: nessun ruolo per le divinità, nessuna forma di antropomorfismo, inferenze comprensibili, controllabili, trasparenti, estrema semplicità delle dinamiche ipotizzate, capacità di rappresentarsi la condizione della terra nel suo insieme e quindi una serie di relazioni sistemiche e, infine, esemplare capacità di prescindere dal fatto di vivere in un angolo qualsiasi dell'area mediterranea.

Si delinea, con ciò, un'analogia con Omero e Platone che forse merita di essere riportata: all'inizio dell'Odissea Zeus presiede il „concilio degli dei” e lamenta che gli uomini attribuiscano agli dei delle colpe per poi discolparsi in malafede. In questo modo prende forma un memorabile rovesciamento: si guarda al mondo umano con gli occhi degli dei. Qualcosa del genere ha fatto anche Platone quando ha introdotto la nozione di imitazione o partecipazione al mondo delle idee, oppure di giudizio ultramondano. Orbene, anche Anassimandro è stato capace di guardare all'entità fisica „terra” dal di fuori, da lontano, fino a vederla e descriverla, ma non è la stessa cosa. Lo dimostra il fatto che almeno noi possiamo agevolmente offrire la resa grafica di ciò che egli ha ,visto' e ci ha insegnato a ,vedere'. Si tratta di una figura di questo tipo:

Non sappiamo se egli fu capace di elaborare un disegno analogo, ma l'osservazione dei rocchi di colonna da montare l'uno sull'altro (e ancora da scanalare) sicuramente fece parte delle esperienze possibili per lui e per non pochi suoi contemporanei, ai quali sicuramente poteva accadere di osservare questi rocchi da una distanza apprezzabile e, dunque, in una forma molto prossima a quella qui suggerita.

\section{L'ideazione del proto-pinax e di una AU con cui ,misurare' perfino il cosmo}

Ma davvero tutto è così semplice? Sicuro che dietro la pretesa di stabilire l'altezza del cilindro terrestre non si nasconda qualcos'altro? Un primo punto su cui riflettere è certamente l'individuazione (e l'immediato impiego) di una non equivoca unità di misura: il supposto diametro terrestre. Questa nozione, a sua volta, appare indissolubilmente 
associata alle caratteristiche del proto-pinax, la cui ideazione e realizzazione è concordemente attribuita ad Anassimandro.

Sull'argomento abbiamo la testimonianza concorde di molte fonti: Agatemero, nelle prime righe della sua Geographia, scrive che Anassimandro «fu il primo ad osare la resa grafica del mondo su tavola» (prōtos etolmēse tēn oikoumenēn en pinaki grapsai: 12A6 DK $=\operatorname{Ar} 47$ W.) mentre Ecateo sarebbe stato «più preciso» (diēkribōsen); Strabone riferisce che, secondo Eratostene, fu lui il primo a «pubblicare una carta geografica» (ekdounai prōton geōgraphikon pinaka: $12 \mathrm{~A} 6 \mathrm{DK}=\mathrm{Ar} 32 \mathrm{~W}$.); Diogene Laerzio che Anassimandro «per primo realizzò la resa grafica del perimetro della terra e del mare» (gês kai thalassēs perimetron prōtos egrapsen ${ }^{13}: 12 \mathrm{~A} 1 \mathrm{DK}=\mathrm{Ar} 92 \mathrm{~W}$.); negli scolii anonimi all'opera geografica di Dionisio il Periegeta si legge: «chi furono i primi a rappresentare l'ecumene in una tavola? Anassimandro» (tines proteron en pinaki tēn oikoumenēn egrapsan? Ar 141 W.). In materia è dunque disponibile una testimonianza esplicita ed unanime, resa anzitutto da autorevoli cultori del sapere geografico.

Due secoli dopo Anassimandro, Aristotele non esitò ad assumere che i dati raccolti nelle carte geografiche si basano su luoghi visti personalmente dal cartografo o su dati desunti da chi li ha effettivamente visti (Meteor. I 13, 350a14-18): un bel riconoscimento della loro qualità, mediamente buona. $\mathrm{E}$ «poiché Aristotele parla delle carte prodotte ai suoi tempi in termini quasi identici a quelli usati da Erodoto, ne inferiamo che fino alla seconda metà del IV secolo non furono introdotti grandi cambiamenti» rispetto al modello prodotto da Anassimandro e ben presto perfezionato da Ecateo (Heidel 1937: 131). Sempre Aristotele, nei Meteorologica, ha occasione di osservare che la larghezza della terra «dalle colonne d'Ercole all'India» dovrebbe essere molto superiore alla sua profondità («dall'Etiopia alla Scizia») e suggerisce che tra le due misure sussista un rapporto non da 1 a 1 (come suggerivano ancora le ,carte'), ma da 5 a 3 (II 5, 362b20-27) $)^{14}$. Da ciò si deduce che il modello fissato da Anassimandro, e fondato sull'idea omerica di Oceano, ha fatto testo, nel senso che Ecateo, e poi altri non solo fino ai tempi di Erodoto, ma addirittura fino ai tempi di Aristotele, si mantennero mediamente fedeli allo schema delineato nel proto-pinax, salvo ad offrire ogni volta qualche dettaglio in più e a ricercare una maggior precisione nella rappresentazione di singoli dettagli. Queste circostanze, sostenute dalla notizia che Ecateo ha solo migliorato la ,carta' di Anassimandro, incoraggiano a pensare che il proto-pinax presentasse, nella cornice del fiume Oceano, una prima rappresentazione, mediamente credibile, dell'intera area mediterranea, grosso modo dalle Colonne d'Ercole al Caucaso (anzi, forse al Caspio), probabilmente con indicazione di un certo numero di toponimi e sicuramente con l'aggiunta di terre ignote, situate

\footnotetext{
${ }^{13}$ L'espressione potrebbe alludere tanto ai margini esterni della terra quanto alla linea di separazione tra i mari e le terre.

14 Anche Erodoto (IV 36.2) aveva segnalato l'insostenibilità del modello circolare, adducendo che l'Asia dovrebbe essere considerevolmente più estesa dell'Europa. Ciò conferma che Aristotele continua a ragionare come Erodoto.
} 
a grande distanza, a nord e a sud dell'area mediterranea e introdotte solo per poter conferire all'insieme la forma circolare, cioè per poter ,riempire' il cerchio.

Riconoscere ad Anassimandro un simile primato contrasta, almeno in prima approssimazione, con la diffusione della rappresentazione diagrammatica di piccoli territori (una casa, le mura della città, un tratto di percorso non rettilineo del fiume et sim.) tanto in Egitto quanto in Mesopotamia, dove quella fu una pratica millenaria. Non risulta però che, fuori dal circuito ellenico, sia stata tentata anche la rappresentazione del mondo o almeno di territori molto grandi; viceversa in ambiente ellenico si ebbero i ,mappamondi' ma non anche le carte settoriali. Inducono a pensarlo le dichiarazioni sopra riportate così come un passo di Eliano (ca. 200 d.C.) in cui viene narrato il seguente aneddoto su Socrate e Alcibiade: quest'ultimo venne invitato ad individuare le sue proprietà terriere a partire da un ,pannello' nel quale era rappresentata tutta la terra (pinakion echōn gēs periodon: $v$.h. III 28), cioè una ,carta' di tipo anassimandreo in cui peraltro sarebbe stato possibile, quanto meno, individuare e riconoscere l'Attica. Analogamente il mondo degli agrimensori romani si direbbe più vicino alla cartografia egizia e mesopotamica che non a quella greca ${ }^{15}$. Pertanto ci sono le condizioni per parlare di due percorsi indipendenti e che non si incontrarono. Queste circostanze confermano che, con Anassimandro, si ebbe un nuovo inizio, autonomo dalle esperienze di altri popoli.

Ora un nuovo inizio, un inizio che parta virtualmente da zero, non è descrivibile solo in termini di ardimento e genialità. Un ruolo non meno grande l'ha avuto il delinearsi di una disciplina, l'affiorare di un criterio, l'oggettività di un'impresa alla quale anche altri potranno liberamente e utilmente dedicarsi attendendosi alla stessa metodologia. Quanto poi al percorso che ha condotto a un risultato così importante, non possiamo non pensare che esso si fondi sulla progressiva aggregazione delle informazioni connesse alla colonizzazione, tanto più sapendo che, se Rodii e Focei si spinsero fino al regno di Tartesso (odierna Andalusia), dunque probabilmente fin oltre le Colonne d'Ercole, proprio i concittadini di Anassimandro seppero colonizzare piuttosto capillarmente le coste del Mar Nero, incluso il suo margine più orientale. Ma quali poterono essere i passaggi successivi? Sicuramente non furono pochi.

Le informazioni fornite dai viaggiatori erano tali da stravolgere il modello omeri$\operatorname{co}^{16}$ in molti modi: il Ponto è un mare immenso ma chiuso; anche il suo limite orientale è noto; l'Oceano, se c'è, deve passare più lontano, oltre i monti situati nell'entroterra del margine orientale del Ponto (forse si seppe che oltre quei monti si apriva un altro grande mare, il Caspio); anche il vasto mare che circonda l'isola di Cipro è un mare chiuso; un altro mare si affaccia non lontano dalle foci del Nilo, ma non c'è comunicazione tra quel mare e i ,nostri' mari; a occidente c'è un altro mare chiuso, l'Adriatico; ancora più

15 Cf. Harley \& Woodward (1987: 130): «the extent to which the early Greeks were influenced by such knowledge (the earlier Babylonian or Egyptian cartography) remains a matter for conjecture».

16 Sulla rappresentazione omerica della terra posso segnalare da un lato la Homerische Erdkarte tracciata da Forbiger (1842, vol. I: 4-5) e, dall'altro, il 'mappamondo' che Giovanni Cerri pubblica in questo stesso fascicolo di Peitho. 
a occidente c'è un altro mare immenso con tre grandi isole (Sicilia, Sardegna, Corsica) e altre minori, che ha una sola, piccola apertura nota col nome di Colonne d'Ercole. Di conseguenza tutti questi mari formano un sistema chiuso perché innumerevoli terre lo circondano da tutti i lati, con un solo passaggio verso l'esterno. Se, d'altronde, tutti questi mari sono circondati e delimitati da una ininterrotta successione di terre (quindi anche di insediamenti abitativi) allora (Heilen 2000: 36 ) fu possibile giungere alla conclusione che, in teoria, si potrebbe compiere un giro completo (gēs periodon) delle terre che lambiscono e delimitano il grande mare interno. Basterebbe costeggiare e, partendo dalle Colonne d'Ercole, si arriverebbe di nuovo alle Colonne d'Ercole passando per molte località diverse, inclusa la quasi totalità delle colonie greche. Pertanto l'Oceano non può che passare laddove ciascuna delle terre lambite dal grande mare interno finisce, quindi oltre le Colonne d'Ercole e oltre i monti del Caucaso, molto oltre le terre calde situate nei territori interni di Egitto e Libia e molto oltre le terre fredde situate a settentrione. Quanto poi all'Oceano, siamo conseguentemente obbligati a pensare che esso si trovi a una distanza immensamente maggiore di quanto aveva lasciato intendere Omero ed abbia una lunghezza poco meno che inimmaginabile. Il mondo si stava rivelando - si è appena rivelato - immensamente più grande di come si era comunemente creduto fino a non molti decenni prima, e le nuove dimensioni del mondo stanno al cosiddetto mappamondo omerico proprio come le dimensioni da attribuire alla terra una volta individuata l'esistenza del Pacifico (e compiuto almeno una volta il suo attraversamento) stanno alle dimensioni immaginate da Colombo quando egli pensò e disse di essere arrivato alle Indie o al Catai.

Le conoscenze e i ragionamenti ora riportati figurano tra le precondizioni per poter arrivare a rappresentarsi i tanti territori lontani di cui si cominciava ad avere idea, con l'Oceano all'esterno, il sistema marino al centro, e sconfinate terrae incognitae situate, in prevalenza, a nord e sud. Sappiamo che l'ambiziosissimo proposito di rappresentare il grande mare interno (con penisole ed isole?), alcuni degli insediamenti abitativi che si susseguivano lungo le coste, i territori lontani dal mare e infine l'Oceano che passa al di dà di tutti questi territori fu concepito per primo da Anassimandro. Fu dunque lui, possiamo presumere, ad insegnare come era fatto il mondo e quanto fosse più grande del previsto (egli fu dunque il Cristoforo Colombo o, meglio, l'Amerigo Vespucci del suo tempo?). In effetti, questo genere di ragionamenti non fu fatto da altri prima di Anassimandro, mentre subito dopo ciò si tradusse in un insieme di conoscenze acquisite e non più problematiche.

Il passaggio successivo fu non l'aspirazione a, ma l'effettiva elaborazione di una rappresentazione congrua, altra impresa di rilievo. Qui sembra collocarsi, infatti, quella che noi chiamiamo „rappresentazione diagrammatica”: una rappresentazione di terre e mari che permette all'osservatore non solo di figurarsi mentalmente, ma di vedere davanti a sé e con i propri occhi una intera serie di territori, come se li osservasse da un'altezza di gran lunga superiore alle possibilità umane: da un non generico punto di vista virtuale. Questo tipo di rappresentazione, si noti, ha anche l'ulteriore caratteristica di comportare una riduzione ragionevole della gamma dei dati da rilevare: il solo 
profilo orizzontale delle coste (non le alture e gli isolotti, non i dettagli del porto e della foce, non le piramidi e altri edifici monumentali), con ricerca di forme e proporzioni plausibili da assegnare a tutti questi spazi grandissimi, in primis l'immenso mare orientale (il Ponto) e il mare occidentale, anch'esso immenso. In altre parole, prese forma (venne ideata e realizzata) una modalità semplificata e coerente di disporre con ordine una grande quantità di idee necessariamente approssimative (forse, spiacevolmente approssimative) su coste, grandi isole, forme dei mari più grandi, insediamenti abitativi e, forse, i sistemi montuosi di cui si sapeva almeno qualcosa, e tutto questo non a fini di intrattenimento ma con finalità conoscitive.

Ci sono pertanto le condizioni per parlare di invenzione di un nuovo medium (Gehrke 1998: 171) caratterizzato, anzitutto, dalla possibilità di osservare i territori come se l'osservatore li guardasse da un'altezza di gran lunga superiore alle possibilità umane (il punto di vista virtuale in grado di abbracciare l'intera superficie terrestre, Oceano periferico incluso).

Ma il nuovo medium ha subito dimostrato di avere anche l'impagabile pregio di far passare la scrittura alfabetica, già relativamente accessibile, per un codice che al confronto è incredibilmente più complicato (ricordo soltanto che la scrittura non è analogica, la rappresentazione diagrammatica si).

L'avvenuta delineazione del proto-pinax ha istituito, in terzo luogo, la non meno inedita possibilità di arrivare a dire „qui c'è la Sicilia, qui Creta, là il Nilo, questa è l'isola di Rodi etc." anche senza aver mai compiuto lunghi viaggi. Dobbiamo immaginare, io credo, l'emozione provata al primo formarsi di un sapere improvvisamente divenuto così facilmente accessibile. Si aggiunga che, malgrado fosse, all'epoca, impensabile misurare le grandi distanze (salvo che in termini di giornate di viaggio ${ }^{17}$ ), il proto-pinax permetteva di farsi almeno una prima idea delle distanze, cioè di figurarsele, dando luogo a figure, forme, proporzioni e comparazioni certamente approssimative, ma pur sempre pertinenti ${ }^{18}$.

Con ciò raggiungiamo una saldatura importante tra le notizie: Anassimandro ha saputo ideare e realizzare il proto-pinax non solo perché ebbe l'idea della rappresentazione diagrammatica, ma anche perché riuscì a rappresentarsi mentalmente l'intero bacino del Mediterraneo, e così pure a ,spostare' l'Oceano immensamente più lontano di dove l'aveva collocato Omero, nonché a rappresentarselo come un bacino idrico molto, molto più lungo ${ }^{19}$. Se dunque egli pervenne a identificare l'area mediterranea e vasti entroterra

\footnotetext{
${ }^{17}$ Le fonti ci dicono che Scilace di Carianda e poi Erodoto istituirono (o provarono a istituire) una proporzione fissa tra giornate di navigazione e stadi. Ma questo è avvenuto più di mezzo secolo dopo e, rispettivamente, quasi un secolo e mezzo dopo!

${ }^{18}$ Es. provare a dire che la lunghezza dell'isola di Creta è approssimativamente pari alla distanza tra Mileto e Naxos. - Giunge il momento di far presente che ho qui sintetizzato quella più articolata riflessione intorno al proto-pinax che figura in Rossetti (2013c).

19 Constato che gli storici della geografia antica (es. Harley e Woodward 1987; Gehrke 1998) sono tra coloro che hanno avuto difficoltà a riconoscere la portata dell'opera di Anassimandro.
} 
con l'ecumene (il ,mondo'), si può capire che egli abbia potuto anche concepire i ,confini' del mondo ed elaborare anche la nozione di estensione massima della superficie terrestre, così da rappresentarsi la distanza da un estremo all'altro (il diametro) senza aver bisogno di provare a quantificarla. Si può quindi capire che egli ne abbia potuto fare anche la prima Astronomic Unit (AU) della storia, ma a questo punto entrano in gioco anche le risultanze della riflessione sui limiti inferiori della terra. Due percorsi indipendenti, due conclusioni pervengono a saldarsi e rendere possibili le ulteriori inferenze che sono state schematicamente descritte nel precedente $\$ 4$. Il risultato è stato di ampliare ancora - e in misura impressionante - il cono osservativo, fino a rappresentarsi la terra vista, come dicevo, di tre quarti.

Pertanto, è ragionevole presumere che, dietro all'indicazione di $1 / 3$, ci sia anche un'altra acquisizione, un'altra assoluta primizia, non meno memorabile di quelle già passate in rassegna: l'avvenuta elaborazione della nozione di unità di misura astronomica pari al supposto diametro terrestre (definito dalla forma circolare dell'Oceano e, più precisamente, dai suoi margini esterni) ${ }^{20}$ che, a sua volta, poté essere pensata solo a partire dall'avvenuta ideazione e realizzazione del proto-pinax. Il potente - e, ripeto, disciplinatissimo - sguardo verso l'ignoto, di cui Anassimandro ha così dato prova, non presuppone unicamente l'ambizione di tentare la resa grafica di luoghi non visti (e unicamente in base all'intelligente collazione dei dati riferiti da una molteplicità di naviganti). Presuppone anche la decisione di non limitarsi a concepire la terra come l'insieme costituito dalle terre e dai mari tra i quali ci muoviamo o ci potremmo muovere, inclusi i luoghi che ancora non si conoscono (ma che confidiamo di raggiungere in futuro, almeno nel senso che qualcuno li potrebbe raggiungere in fuuturo: essa stessa una conquista memorabile). E inoltre presuppone la decisione di procedere immediatamente a ,pensare' la distanza massima o diametro, cioè a rappresentarsela ancor prima di conoscerla, e ben sapendo di non essere in grado di misurarla in qualche modo.

L'ardimento è consistito, presumo, nell'accedere immediatamente all'idea che la distanza massima sia una nozione utilizzabile, anche se nota solo con innumerevoli (e, a loro modo, immense) approssimazioni ${ }^{21}$, e utilizzabile per farci un'idea - almeno un'idea! - delle distanze cosmiche, cioè di quelle grandezze che non sapremo mai propriamente osservare e comparare, tanto meno misurare. Per il fatto di applicare al cosmo quelle misurazioni che erano rese possibili dalla sua ,carta ${ }^{\prime 22}$ possiamo ben dire che, una volta realizzato il proto-pinax, Anassimandro ha avuto la prontezza di intuire immedia-

${ }^{20}$ Se non sbaglio, le misure di Anassimandro sono state associate per la prima volta alla nozione di AU in White (2008).

${ }^{21}$ In teoria si può ipotizzare il seguente ragionamento: il diametros terrestre dovrebbe essere pari, supponiamo, a dieci, quindici, venti volte la distanza da Creta alla Sicilia, oppure da Creta a Corfù. Simili misurazioni non avrebbero avuto alcuna pretesa di essere scambiate per effettive, ma avrebbero ugualmente soddisfatto il bisogno di orientarsi.

${ }^{22}$ Cf. nota 17. Le due situazioni non sono molto differenti, perché in nessuno dei due casi si perviene a sapere ma, in compenso, si riesce a comparare (1/3, oppure $9,18,27$ volte) e dunque a stimare, a farsi un'idea di ciò che altrimenti non sapremmo in alcun modo collocare nello spazio, limitare e circoscrivere. 
tamente questa ulteriore (e impensata) potenzialità metrologica. Del resto il suo sapere è complessivamente caratterizzato dalla propensione a compiere non uno, ma innumerevoli (non però stravaganti, ma - ripeto - disciplinati, giudiziosi e altamente benemeriti) salti nel buio.

\section{Altre tessere, non così ben stabilite, del sapere cosmologico di Anassimandro}

Fino a questo punto è stato possibile raccordare informazioni assai ben protette da dubbi sul loro contenuto informativo così come sulla loro ascrivibilità ad Anassimandro. Giunge ora il momento di passare in rassegna un gruppo di informazioni molto meno sicure, che è prudente non porre sullo stesso piano di quelle fin qui richiamate (né di altre che verranno esaminate più avanti, nei $\$ 8-11)$.

Tale è il caso della supposta dislocazione dei corpi celesti nello spazio in modo tale da formare delle fasce concentriche. Sin dai tempi del Tannery (1887), e poi in alcuni lavori recenti ${ }^{23}$, numerosi specialisti hanno ipotizzato che Anassimandro, stimolato dalla possibilità di delineare un intero sistema di distanze dalla terra, abbia effettivamente indicato la serie completa delle distanze spaziali assegnate, con ulteriori precisazioni intorno alla forma attribuita a una (o, forse, tre) immense strutture circolari invisibili a occhio nudo ed altri dettagli. In questo caso, tuttavia, la base inferenziale primaria è costituita dalla mera discrepanza tra le fonti: in due passi di Eusebio (=pseudo-Plutarco) la supposta distanza del sole viene indicata ora in 28 e ora in 27 diametri terrestri, mentre Ippolito parla di 27 diametri e Stobeo di 28; inoltre il medesimo Stobeo indica in 19 diametri la distanza della luna ( $\operatorname{Ar} 57-58,75.5,150-151 \mathrm{~W}$.). Si è dunque supposto che la discrepanza non si debba sciogliere optando per una sola delle due quantificazioni, ma si debba mantenere intendendo che Anassimandro abbia (a) attribuito 9 AU alla distanza terra-stelle, altre 9 AU alla distanza stelle-luna e altre 9 AU alla distanza luna-sole; (b) assegnato ad ognuno di questi oggetti spaziali l'ampiezza di $1 \mathrm{AU}$, cosicché le distanze nette sarebbero pari non a 9 ma a $8 \mathrm{AU}$; (c) individuato la distanza massima di $28 \mathrm{AU}$ sommando alla distanza netta ( $8 \times 3$ ) il diametro di terra, stelle, luna e sole (altre quattro AU).

L'ipotesi ${ }^{24}$ è decisamente macchinosa e presuppone la piena attendibilità di ciascuno dei dati disponibili. Ci si può certo spingere ad affermare che i dati offerti dalle fonti sono compatibili con una simile combinazione, ma è molto dubbio che dalle fonti emergano indizi sufficienti, e sufficientemente coerenti, per accreditare una così complessa congettura. Qualche ombra lambisce, del resto, anche i due punti comparativamente più sicuri: da un lato l'assunto che i corpi celesti si dispongano su tre livelli a distanze crescenti,

${ }^{23}$ Ricordo in particolare Naddaf (2001), Hahn (2001 e 2010) e il recente Corre (2013).

${ }^{24}$ In realtà non è l'unica ipotesi. KRS (1983: 136) proponeva di sommare alle 27 AU il diametro terrestre anziché il diametro solare. Altre variazioni sul tema (non il raggio ma il diametro della struttura anulare, non il diametro ma la circonferenza, una possibile correzione del valore da assegnare a $\pi . .$. ) vengono ora prospettate - e con singolare abbondanza - in Corre (2013). 
dall'altro forma e dimensioni della fascia cosmica che dovrebbe, contenere' tutte le stelle. In queste condizioni non è più prudente riconoscere che, sull'argomento, si possono solo avanzare labili congetture? ${ }^{25}$

Considerazioni analoghe si applicano alle ipotesi sull'esatta configurazione delle strutture circolari che ,trasportano' il sole e la luna (nonché, si è supposto, le stelle). Le fonti ci dicono che Anassimandro ha concepito, attorno alla terra, tre, due o almeno un grande cerchio: cerchi invisibili, cioè trasparenti. Il sole, poi, starebbe all'interno di questa struttura e si affaccerebbe da una sorta di grande finestra. Gli studiosi si sono variamente affaticati nel tentativo di far emergere un senso plausibile dalle non poche informazioni che, sull'argomento, sono giunte fino a noi ma, date le tenaci incertezze che questa contorta materia presenta (tra l'altro, all'epoca non si aveva certo idea di sostanze solide che fossero perfettamente trasparenti malgrado le grandi dimensioni), credo che sia preferibile, ancora una volta, prendere atto della difficoltà e sospendere il giudizio.

Ricordo inoltre che, secondo alcuni studiosi, l'espressione kai sphairan kateskeuase (Diog. Laert. II 2) si dovrebbe interpretare nel senso che Anassimandro seppe realizzare - anzi, realizzò - perfino un proto-modello astronomico su scala, con la terra al centro e i corpi celesti collocati in tre cerchi concentrici. Nemmeno questa tesi, rilanciata in particolare dal Betegh (2002, spec. p. 405: «Son système cosmologique se prête facilement à une modélisation tridimensionnelle»), è però priva di serie complicazioni: basti considerare che l'insieme, costituito da un cilindro e da tre grandi cerchi o ruote, non ha motivo di dare luogo a una sfera e che, se i grandi cerchi in miniatura non possono non essere disposti su un piano orizzontale, il cilindro terrestre collocato al centro dovrebbe essere non solo piccolissimo (per rispettare le proporzioni: 1/27), ma anche disposto con l'asse in posizione approssimativamente orizzontale, nel qual caso la superficie piana corrispondente alla terra a noi nota e da noi abitata dovrebbe, al pari della superficie opposta (antipodi), trovarsi in posizione verticale. Così stando le cose, ha senso chiedersi: non sarà troppo presumere anche questo? Del resto, non abbiamo motivo di dare per certo che le congetture sulla più precisa disposizione dei corpi celesti nello spazio abbiano avuto un ruolo centrale e qualificante nell'insegnamento di Anassimandro.

Ancor più dubbia deve considerarsi la doxa concernente l'inclinazione dell'eclittica. Scrive Plinio (12A5 DK = Ar 40 W.) che obliquitatem eius (dello zodiaco) intellexisse ... Anaximander Milesius traditur primus Olympiade quinquagesima octava. A sua volta Stobeo (riconducibile ad Aezio: $12 \mathrm{~A} 22 \mathrm{DK}=\mathrm{Ar} 151$ W.) scrive che, secondo Anassimandro, il kyklos della luna, al pari di quello solare, giace in posizione obliqua (keitai loxon). La tradizione esegetica ha a lungo valorizzato questa informazione senza esitare nemmeno (es. Kahn 1960: 88: «Anassimandro deve (must) aver avuto familiarità con l'inclinazione dell'eclittica in rapporto al percorso diurno delle stelle»). Osservo però che c’è una grande differenza tra dichiarare che il supposto percorso circolare di sole e luna

\footnotetext{
25 Questo è stato, in ultima istanza, il punto di vista difeso in Kahn (1960: 62 s. e 88); ed è anche la conclusione raggiunta dal Couprie (2011, cap. 9) mezzo secolo dopo.
} 
è inclinato e teorizzare l'obliquità dello zodiaco. La prima di queste due affermazioni si limita ad aggiungere una constatazione del tutto elementare alla tesi, verosimilmente già accreditata, secondo cui ogni notte sole e luna completano il loro tragitto circolare. Infatti si tratta soltanto di annotare che, come può agevolmente constatare ogni abitante di Mileto e della generalità degli insediamenti ellenici, quel tragitto si colloca in posizione più o meno accentuatamente obliqua. In aggiunta si potrebbe osservare che Anassimandro probabilmente ignora che a Siene, nell'alto Egitto, accade che per un giorno il sole non sia inclinato ${ }^{26}$. Ma Plinio parla di comprensione dell'inclinazione dello zodiaco (che è ben altra cosa), ossia di spiegazione del fenomeno nel presupposto che si tratti di un fenomeno già noto. A supporto di questa sua affermazione egli si limita a indicare un anno preciso (ma alquanto inverosimile: nel 544 a.C. Anassimandro poteva essere già morto), mentre nessun'altra informazione ci aiuta a capire che valore potesse avere, per Anassimandro, questo fenomeno. In terzo luogo, l'informazione fornita da Plinio non è propriamente cumulabile con quella fornita da Stobeo-Aezio sia perché lo zodiaco non è la luna né il sole, sia perché Plinio associa Anassimandro ad un nucleo dottrinale che, nell'astronomia dei tempi di Plinio, era già fermamente stabilito. Pertanto la prudenza esegetica invita, ancora una volta, a considerare che, dietro all'informazione qui offerta, potrebbe anche esserci una mera ricerca di padri nobili non sospettata da Plinio. In conclusione, il poco che sappiamo non autorizza ad attribuire ad Anassimandro un sapere astronomico specifico intorno allo zodiaco o all'eclittica ${ }^{27}$.

Ancora, Stobeo (12A22 DK = Ar 152 W.) attribuisce ad Anassimandro (nonché a Senofane e Beroso) la tesi secondo cui la luna risplende di luce propria. In effetti, se la luna costituisce, al pari di sole e stelle, uno dei derivati del fuoco che si è staccato dalla terra in un passato oltremodo lontano $(\$ 11)$, è logico che sia dotata di luce propria al pari degli altri corpi celesti. Bisognerebbe però capire se Talete ha positivamente insegnato che, quando ha luogo l'eclisse, la luna copre il sole perché è opaca, cioè perché non brilla di luce propria. In tal caso, l'allievo avrebbe rigettato uno specifico insegnamento del maestro e la notizia sarebbe significativa. Ma su come Talete si spiegò l'opacità della luna nel novilunio navighiamo nel buio più totale.

Infine la tesi dell'infinità dei mondi, cioè della infinita quantità di mondi che si susseguirebbero nel tempo o coesisterebbero nello spazio. Come ho già ricordato $\left(\$_{3}\right)$, che in Anassimandro siano state rintracciate affermazioni in grado di far pensare ad una qualche pluralità di mondi è una eventualità da considerare. Nondimeno i dubbi permangono. D'altronde, la tesi della pluralità non sembra aver attitudine a modificare il resto (cioè

${ }^{26}$ Che Anassimandro lo ignori mi pare del tutto verosimile: perché mai dovrebbe aver prestato attenzione a un dettaglio di cui tace lo stesso Erodoto, nonostante questi abbia molto da dire proprio sul conto di SieneElefantina? La particolarità del sole a picco per un giorno all'anno in località a sud di Siene (ma non troppo lontana da Siene) fu forse notata già prima che se ne occupasse Eratostene, ma questo è tutto. Pertanto non solo il silenzio di Erodoto ma anche il passo di Stobeo-Aezio depone contro la tesi di Naddaf (2005: 100) e Couprie (2011: 134 s.), secondo cui è difficile credere che Anassimandro non avesse notizia di ciò che accade a Siene in occasione del sostizio estivo.

${ }^{27}$ Così anche Dúhrsen (2013: 305). 
il nucleo centrale, la struttura portante) degli insegnamenti di Anassimandro. Se dunque fu tesi positivamente sostenuta, si tratterebbe pur sempre di un dettaglio marginale.

Riepilogando: possiamo e dobbiamo certamente riconoscere ad Anassimandro il merito di aver saputo offrire - e deve essersi trattato, di nuovo, di una storica primizia, di una felice intuizione - anche un primo modo, avventuroso ma, insisto, non certo stravagante ${ }^{28}$, di valorizzare una grandezza - più propriamente una distanza - già straordinariamente grande e certo non misurabile (ma almeno concepibile), il supposto diametro terrestre, per rappresentarsi le distanze spaziali (quindi le misure dell'extraterrestre, l'irriducibilmente lontano). Infatti poté apparire sensato e, a suo modo, istruttivo parlare di distanze pari ad alcune decine di diametri terrestri. Lo comprendiamo molto bene noi che non ci siamo ancora stancati di meravigliarci di fronte all'inabbracciabile immensità di un macrocosmo che ci dicono essere sempre più grande di come ci era stato indicato appena un anno prima. Così facendo, Anassimandro ha saputo operare una oltremodo drastica - e creativa - riduzione della complessità ${ }^{29}$, mentre ci ha reso partecipi dell'emozione che si prova nel superare e di molto i supposti limiti della condizione umana, tanto da poter almeno avviare la misurazione di grandezze spaziali che, all'epoca, dovevano essere ritenute smisurate per definizione.

Ma gli altri punti ora passati in rapida rassegna non si possono mettere sullo stesso piano della straordinaria lezione di astronomia di cui siamo debitori ad Anassimandro, della messa a punto di un medium comunicazionale così importante come la carta geografica e di altri apporti, come la capacità di pensare ad una terra che si trasforma nel lungo periodo (cf. $\$ 8-9$ ). Da una parte c'è un grande e multiforme ktēma eis aiei, dall'altra non abbiamo solo una serie di informazioni molto meno affidabili, ma anche nuclei dottrinali la cui rilevanza dovrebbe essere stata incomparabilmente minore.

Ciò premesso, prenderò ora in considerazione altre tessere erratiche aventi per oggetto delle misurazioni.

(a) Plinio riferisce che sulla data del ,tramonto' mattutino delle Pleiadi sono state manifestate opinioni diverse: Esiodo avrebbe detto che le Pleiadi tramontano allorché, finisce' l'equinozio di autunno, Talete che tramontano 25 giorni dopo, Anassimandro che tramontano 31 giorni dopo e altri che tramontano 44048 giorni dopo $(11 \mathrm{~A} 18 \mathrm{DK}=12 \mathrm{~A} 2 \mathrm{O}$ $\mathrm{DK}=\operatorname{Th} 106$ e Ar 44 W.). La notizia è significativa, se non altro perché ci dice che Esiodo mostra di aver trattato l'equinozio (di autunno) non come un evento puntuale ma come un evento che si protrae per giorni o settimane, mentre Talete avrebbe pensato per primo ad una data precisa, così da poter poi stabilire quanti giorni dopo l'equinozio ha luogo il ,tramonto’ delle Pleiadi. Questa ed altre circostanze permettono di capire che, se Talete

${ }^{28}$ In effetti non capisxco come si possa scrivere (KRS 1983: 137), che «It is plain that much of Anaximander's astronomy is speculative and aprioristic». Aprioristiche non sono molte ma, semmai, una minoranza di tessere, forse perfino secondarie.

29 Saper dire, sia pure solo in prima approssimazione, quanto sono lontani degli oggetti lontanissimi ottiene fra l'altro di svuotare gli astri del sovraccarico mitologico che, all'epoca, notoriamente gravava su di loro. 
condusse specifiche ricerche volte a datare con precisione proprio l'equinozio autunnale ${ }^{30}$, il discepolo si permise di ripetere le misurazioni, allo scopo di precisare ulteriormente la distanza tra le due date, e non esitò a contraddire il suo maestro.

(b) Sembra che Anassimandro abbia anche provato a stimare la grandezza effettiva della luna (non esattamente di sole e luna, come invece viene riferito) grazie alla stima di quanto dura la congiunzione della luna con i pianeti Mercurio e Venere (metaparabolē di Hermes e Afrodite: Simplicio in 12A19 DK = Ar 185 W.). L'idea è certamente creativa ma, anche limitando il riferimento alla luna e anche concedendo che Anassimandro potesse aver avuto notizia dell'anomalo status di alcune stelle ,errabonde', si direbbe destinata a rimanere un vano desiderio, dato che la combinazione del valore ricercato (quanto è grande la luna) con la durata dell'oscuramento delle due stelle errabonde non sembra per nulla promettente (senza considerare poi la difficoltà di quantificare i pochi minuti dell'intervallo temporale che egli si sarebbe proposto di prendere in considerazione). Si osserva inoltre l'apparente assenza di nessi tra questo particolare dato e l'insieme del sapere anassimandreo sui corpi celesti.

(c) Ci viene anche riferito che Anassimandro inventò lo gnomone e lo ,esportò' a Sparta (Diog. Laert. II 1 e altre fonti). Che lo strumento possa essere stato ideato da lui è ben poco verosimile; è invece possibile che egli si sia specializzato nel suo impiego e che ne abbia installato uno a Sparta ${ }^{31}$. Che lo gnomone possa aver interagito con tessere importanti del sapere di Anassimandro è stato sostenuto di recente dal Corre (2010), il quale immagina che questo antico intellettuale sia stato capace di rilevare che, nel corso dell'anno, la sua ombra traccia una curva concava, poi rettilinea, poi convessa, poi di nuovo rettilinea etc. e che tanto sarebbe bastato per individuare con apprezzabile precisione la data di solstizi ed equinozi. La sua congettura si fonda sulla mera possibilità che Anassimandro sia stato capace di notare tutto ciò, ma l'inferenza non è per nulla ovvia. Qui mi basti osservare che bisogna prima sospettare un particolare andamento delle curve e poi adoperarsi a registrarlo con cura durante molti mesi, per più anni e con crescente precisione (altrimenti non si arriverebbe a fissare delle date con questo solo strumento $^{32}$ ). Inoltre è ragionevole pensare che una simile scoperta avrebbe fatto non meno scalpore della misurazione dell'ampiezza angolare del sole ad opera di Talete ${ }^{33}$ e avrebbe forse cambiato la vita ad Anassimandro. Invece il suo insegnamento, per come

30 Cf. Rossetti (2012).

${ }^{31}$ Corre (2010, nota a p. 15), opportunamente segnala che «l'opération aurait pu se faire sans changement des repères au sol, puisque Sparte est à la même latitude que Milet, à très peu près».

32 Per poter affermare che l'equinozio si è verificato ieri e non due giorni fa si richiederebbe, invero, una rilevazione così precisa da richiedere apparati di registrazione dell'ombra troppo precisi per le capacità tecnologiche e organizzative dell'epoca: creazione di un ambiente di rilevazione da preservare con il più grande scrupolo, assoluta stabilità dell'asta e del piano di rilevazione durante anni, asta appuntita con molta cura, alta qualità degli strumenti di tracciamento della curva - o della retta - e adeguata formazione del personale addetto.

${ }^{33}$ Ricordo che, se Anassimandro pretese di correggere il dato fornito da Talete e, due secoli dopo, Eudosso si preoccupò di retificarlo ancora una volta, nulla viene riferito sulla speciale risorsa offerta dallo gnomone allo scopo di fissare la data dell'equinozio. 
lo perveniamo a conoscere noi a partire dalla documentazione disponibile, non è orientato a misurare, ma a capire come sono conformati i territori, dove all'incirca si trova il Nilo, quale dovrebbe essere la forma della terra, quali le verosimili macro-distanze, quale la macro-storia della terra etc.

Questa ed altre tessere sarebbero comunque marginali rispetto al sapere di Anassimandro sulla forma dell'area mediterranea e della terra nel suo complesso, nonché sui corpi celesti (percorso e distanze).

\section{Il cielo sopra di noi}

Chiusa questa parentesi (che era però doverosa), propongo ora di passare ad un altro punto spesso relegato tra le notizie di scarso interesse, la meteorologia di Anassimandro. Sappiamo bene che il cielo non è fatto solo di astri lontani ma anche di nubi, piogge e fenomeni correlati, e non ignoriamo che dare un senso a questo tipo di ,imprevisti' ha costituito una più che apprezzabile sfida per i presocratici. Bene, anche in questo campo Anassimandro sembra essere stato un apripista.

Seneca con particolare nitidezza, ma anche lo pseudo-Plutarco e Ippolito (più altre fonti secondarie: $12 \mathrm{~A} 23 \mathrm{DK}=\mathrm{Ar} 38,63,75.7,227$ e $267 \mathrm{~W}$.) convergono nel mostrare che Anassimandro si distinse per aver saputo offrire una spiegazione unitaria dei fenomeni meteorologici. Egli omnia ad spiritum rettulit, dice Seneca, e spiega che i tuoni si producono quando le nubi si rompono e che la loro diversità (apparente) dipende dal vento. Se accade di udire dei tuoni con il cielo sereno vuol dire, egli prosegue, che il vento è comunque riuscito a lacerare l'aria; se si odono tuoni ma non si vedono fulmini, vuol dire che il vento è debole e non produce effetti particolarmente vistosi; il lampo è un fulmine attenuato, mentre il fulmine ci parla di uno squarcio traumatico, violento nelle nubi. Pseudo Plutarco precisa che a produrre gli effetti più spettacolari sono le nubi scure.

Il tratto saliente di un simile insieme è ravvisabile nel consapevole proposito di ricondurre ad unum una molteplicità di fenomeni e, naturalmente, nella determinazione con cui viene proposta una spiegazione che non chiama in causa a nessun titolo Zeus. Ogni nube somiglia ad un immenso otre di origine animale agitato dal vento e si può capire che di tanto in tanto si generi un rumoroso attrito tra nubi, oppure che l'una o l'altra si rompa in modo più o meno violento, producendo altro rumore e, talvolta, lampi luminosi, ma non sono nient'altro. Il perché di tuoni e fulmini è facile da capire. Dietro non c'è nessun arcano, e in particolare nessuna forza ingovernabile, nessuno Zeus. Una rassicurante semplicità avvolge tutti questi fenomeni.

Molto significativo è anche un ulteriore dettaglio, riferito da Ippolito: «Le piogge si formano dal vapore che viene fatto salire dalla terra verso l'alto ad opera del sole, $e k<t \bar{s}$ atmidos > tês ek gès huph' hèlion anadidomenēs». Qui viene inequivocabilmente delineato il ciclo delle acque, sul quale si è poi notoriamente soffermato Senofane nel fr. $30 \mathrm{DK}$. Il chiarimento offerto in questo caso da Anassimandro ha il non lieve pregio di apparte- 
nere alla categoria delle scoperte fatte una volta per tutte e subito diventate patrimonio inalienabile dell'umanità (al pari della ruota o del pane lievitato, dello specchio o perché no, del mappamondo). Certo, quella di Ippolito è una voce isolata, ma la notizia deve dirsi pur sempre significativa e priva di specifici indizi di inverosimiglianza. Non è sicura al $100 \%$, ma è pur sempre una informazione da ritenere.

\section{Dai grandi spazi ai grandi tempi: il passato remotissimo della terra. Anassi- mandro evoluzionista}

Indizi non controversi invitano a presumere che il panorama degli insegnamenti di Anassimandro intorno alle relazioni spaziali e ad alcuni fenomeni che hanno luogo in cielo, considerati a prescindere dalla variabile temporale, costituisca una grande, memorabile sub-trattazione, ma non più di questo, dunque nemmeno $i l$ nucleo centrale del suo sapere. Infatti anche un altro e non meno memorabile ,capitolo' concorre a costituire l'insieme, quello che concerne i tempi lunghi della formazione e delle trasformazioni che hanno interessato, interessano e continueranno a interessare la terra, se non il mondo nel suo insieme. Il tema dei grandi spazi si direbbe dunque complementare al tema dei ,tempi lunghi' e le due aree di ricerca, i due sottosistemi di congetture parrebbero configurarsi approssimativamente come le due metà dell'intero, o almeno come le due macro-aree che, pur non esaurendo l'intero, ne sono senza alcun dubbio magna pars.

Giunge dunque il momento di concentrare l'attenzione sui ,tempi lunghi'. A tale scopo, propongo di procedere dal passato relativamente prossimo al passato più remoto. Infatti gli eventi che Anassimandro ha provato a collocare a ritroso nel tempo sembrano disporsi con molta naturalezza lungo una scala cronologica oltremodo dilatata, una scala che sembra arrivare fino uno stadio remotissimo del cosmo.

Quale dunque l'evento più recente su cui Anassimandro risulta essersi soffermato? Non c'è dubbio, si tratta della gestazione dei primi uomini ad opera di qualche grande animale marino. In questo caso la nostra fonte principale, pseudo-Plutarco (citato da Eusebio di Cesarea: $12 \mathrm{~A} 30 \mathrm{DK}=\mathrm{Ar} 101 \mathrm{~W}$.), riferisce tutto un ragionamento: l'uomo non si sarebbe salvato se, all'origine, fosse stato come è adesso; infatti ha bisogno di cure (tithēnēsis) molto prolungate, il che marca la differenza rispetto a tutti gli altri animali, che invece imparano molto presto a nutrirsi da soli. L'informazione compare in forme manifestamente affini anche in Plutarco, Ippolito e Censorino (Ar 45, 75.6 e 90 W.). Oltre alla certezza ed univocità dell'ascrizione, questa notizia ha dunque il raro pregio di preservare il nocciolo di un altro ragionamento di Anassimandro, quello con cui egli ha molto probabilmente illustrato e reso intelligibile la sua congettura, la quale di per sé sarebbe tutt'altro che intuitiva.

Chiaramente qui si parla della comparsa dei primi uomini sulla terra; dunque si pensa già a un passato lontanissimo, quando poté accadere che sulla terra ci fosse 
appena un manipolo di „figli dei pesci”34 (non addirittura il primo uomo). Il ragionamento ha il pregio di sollevare il velo su più di un presupposto: in questo e in altri casi Anassimandro non ipotizza nessun evento miracolistico e non attribuisce alcun ruolo alla divinità. Al contrario, assume come modello la norma che regna nel mondo animale (mammiferi e ovipari di dimensioni relativamente grandi), dove il neonato già gode di un accettabile minimo di autosufficienza; poi constata che l'uomo è un po' differente (gli umani non sanno provvedere a se stessi nei primi 12-15 anni di vita) e ne deduce che deve esserci stata una misura eccezionale per permettere anche agli umani di formarsi ed avere un avvenire. Indirettamente egli si trova a postulare un semi-automatismo per effetto del quale prima o poi anche l'uomo sarebbe dovuto comparire. Egli può così concludere che, all'inizio, qualche altro essere vivente non umano dovette farsi carico della sopravvivenza degli umani durante un periodo straordinariamente lungo e, per esclusione, pensa ad un grande pesce $\mathrm{o}$, più probabilmente, a un certo numero di grandi pesci, i pesci di una certa specie, intendendo che a fine ciclo questi pesci-madre avrebbero avuto cura di andare a deporre il feto sulla riva del mare.

Ciò dimostra che, agli occhi di Anassimandro, l'evento rimane pur sempre ancorato alla normalità degli accadimenti, tanto da venire presentato come un evento non meno naturale di innumerevoli altri. Si tratta, peraltro, di una naturalità che concerne il verificarsi di fatti nuovi di grande portata. Registriamo questo punto con riserva di riprenderlo al termine della rassegna sugli altri fenomeni di tipo evolutivo che Anassimandro mostra di aver identificato.

Un altro evento fondamentale, che pure viene collocato nel tempo, e che è logicamente anteriore (e di molto) alla comparsa dei primi umani, concerne lo stabilirsi di un primo gruppo di animali marini sulla terraferma. Anche in questo caso disponiamo di una congettura ben argomentata (la nostra fonte primaria è Plutarco nello stesso brano): ad un certo punto iniziò la fase di adattamento di alcuni animali marini (di quale specie: i delfini? osservo che, in teoria, si potrebbe pensare anche alle rane o alle tartarughe) alla vita sulla terraferma, andando incontro a trasformazioni rapide, e irreversibili, nelle loro condizioni di vita. Di nuovo, dunque, un evento ,naturale', anch'esso di tipo inequivocabilmente evoluzionistico.

Un terzo evento, col quale si risale molto più indietro, concerne la fase di avvio della generazione dei pesci in un contesto di acqua calda (o meglio: tiepida), verosimilmente molto prima che qualche pesce trovasse il modo di vivere fuori dall'acqua. Ippolito e Censorino (l.c.) riferiscono che l'ambiente favorevole alla formazione dei primi animali marini sarebbe stato caratterizzato da calore e umidità, e l'idea potrebbe anche dipendere da Talete (avrebbe sostenuto che gli esseri viventi si formano in un contesto umido:

\footnotetext{
${ }^{34}$ Plutarco riferisce, invero, che Anassimandro avrebbe anche dichiarato i pesci «padri e madri degli uomini», aggiungendo che il pesce non è buono come cibo per gli umani (verosimilmente, si tratterebbe di un automatismo volto ad esprimere una supposta forma di rispetto per i pesci in genere, in 'ricordo' della gestazione dei primi uomini). Sull'attendibilità della notizia, che non è confermata da altre fonti di informazione, è lecito nutrire dubbi.
} 
così Aristotele in Metaph. I 3, 983a21-27 = 11A12 DK = Th 29 W.) o almeno configurarsi come versione un po' più raffinata di un'idea di Talete.

Questi tre nuclei teorici ci permettono di accedere ad appena tre piccole punte di iceberg. Nondimeno essi delineano, com'è evidente, un'idea complessiva e ben definita del processo di formazione dell'insieme degli esseri animati a partire dai pesci ${ }^{35}$. Infatti escludono tante cose, anzitutto la contemporaneità dei fenomeni. Di conseguenza non sembra azzardato ipotizzare che l'autore pensasse ad un percorso a tappe già relativamente organico, molto diluito nel tempo, in cui ci fosse stato posto dapprima per una terra spoglia e disabitata, poi per la formazione di pesci (e, sulla terraferma, di piante); sarebbe poi seguita la formazione dei primi animali terrestri (e la loro progressiva differenziazione, fino a dar luogo, fra l'altro, agli uccelli), e infine di esseri umani. In questo caso, si noterà, Anassimandro non ha nemmeno provato a quantificare l'arco temporale, data anche l'impossibilità di identificare, come appropriata per i tempi lunghi, una qualunque grandezza temporale che potesse reggere il confronto con la AU.

Le fonti ci parlano di una successione di molteplici eventi creativi dello stesso tipo, che però non hanno un attore identificato e nemmeno una finalità attribuibile: è come se fossero semi-automatici ${ }^{36}$. Guarda caso, anche le moderne scienze della vita danno per acquisito che le specie vegetali e animali abbiano fatto la loro comparsa in tempi diversi mentre rinunciano volentieri a sviluppare una riflessione sul perché della comparsa di sempre nuove specie. Ciò significa che Anassimandro ha saputo elaborare una prima versione (la prima di cui si abbia notizia) dell'evoluzionismo di tipo darwiniano: una versione certamente rozza, ma inequivocabile ${ }^{37}$.

Abbiamo argomenti per non arrivare ad una simile conclusione? Se Cleve (1965: 146-148) aveva parlato di fraintendimenti in relazione a quanto riferisce Plutarco, Barnes ha obiettato che le evidenze disponibili non includono informazioni sulla progressiva diversificazione degli animali: a suo avviso non viene teorizzata «una lunga e graduale alterazione nella forma e nel comportamento delle specie animali in risposta ad un ambiente che si trasforma» e quindi «non c'è traccia di evoluzione» (1979, I 22 s.). In contrario si può osservare che i pesci ,impararono' a vivere in altro modo (metabiōnai: Pseudo-Plutarco) sulla terraferma e persero le squame e i primi uomini rapidamente impararono a nutrirsi pur non avendone la minima idea, tanto da sopravvivere. Questi sono due esempi di adattamento all'ambiente, fors'anche di selezione naturale, perché, se solo alcuni tipi di animali marini ebbero successo nel tentativo di vivere fuori dall'acqua, forse altri tipi non ebbero successo. È dunque il caso di trattare i due esempi come

35 Un dettaglio non trascurabile concerne l'eventualità, non documentata, che Anassimandro abbia saputo far posto, in questa 'storia naturale', anche alla comparsa e successiva proliferazione dei vegetali sulla terraferma.

${ }^{36}$ Infatti avrebbe senso chiedersi: chi ha individuato i pesci idonei ad effettuare la gestazione prolungata? chi ha dato loro delle istruzioni? chi ha detto loro "adesso è tempo di partorire"? chi ha detto loro: "mi raccomando, sulla spiaggia"? chi ha detto loro "bene, adesso basta"? e perché vennero impartiti simili ordini? Silenzio. Anassimandro non sembra essersi posto questo genere di problemi (cf. $\$ 11)$.

37 Così anche Dúhrsen (2013: 306). 
rappresentativi in quanto concernono il buon esito di processi di adattamento particolarmente difficili, più difficili e più rischiosi di altri. Pertanto non è per nulla inverosimile che Anassimandro abbia previsto una molteplicità di processi di trasformazione, anche profondi, che intervengono quando si tratta di riuscire a vivere anche in condizioni ambientali mutate (e meno favorevoli).

Virtualmente certa deve dirsi, inoltre, la distribuzione di tali trasformazioni in tempi molto molto lunghi. Ciò non solo perché è logico pensare che le trasformazioni sono quasi impercettibili solo se hanno luogo nel lungo periodo, ma anche e soprattutto perché, come vedremo tra un momento, Anassimandro ha teorizzato un percorso evolutivo anche per la terra in quanto tale, e questo è certamente pensato come diluito in tempi smisuratamente dilatati.

\section{Il processo di essiccamento della terra}

La serie delle sue ragionate congetture prosegue con un generoso tentativo di immaginare la condizione della terra (e del mondo) prima che si arrivasse all'attuale punto di equilibrio (dunque in un passato molto più remoto) e anche $d o p o$, in un futuro altrettanto remoto. Egli parla di un processo partito da lontano e destinato ad arrivare lontano: in una prima fase la terra era umida (nel senso che costituiva una massa morbida), poi si è innescato un lento processo di essiccazione ad opera del sole, processo che ha determinato sia la formazione di aria e venti, sia la progressiva diversificazione fra terre e mari. Questo processo non riguarda solo il passato, ma anche il futuro in quanto - apprendiamo - si deve prevedere che esso continui, dando luogo al graduale prosciugamento degli stessi mari, forse fino alla loro scomparsa. Il nome di Anassimandro lo fanno Alessandro di Afrodisia e lo pseudo-Plutarco (quindi Aezio), mentre qualche dettaglio aggiungono Aristotele e lo stesso Alessandro (12A27 DK= Ar 8-10, 64 e 66, 84-85 W.). Ci viene dunque segnalato che $i l$ sole ha già trasformato radicalmente la terra e continuerà a trasformarla, per cui la condizione attuale della terra viene declassata, in quest'ottica, ad episodio o fase di una vicenda lunghissima, graduale e ininterrotta.

Il fatto di affermare che la terraferma si è costituita nel corso di un processo lungo e lento, che è tuttora in corso, ci autorizza a pensare che la comparsa di piante, animali ed uomini sia stata opportunamente collocata in una fase favorevole del ciclo, fase che non potrà certo ripetersi quando il prosciugamento dei mari sarà giunto a compimento. Pertanto, che l'uomo potesse comparire solo quando ormai la terra era popolata da molte specie animali e gli animali terrestri solo dopo che i mari si erano riempiti di pesci e la terra cosparsa di erbe, fiori e alberi, tutto questo diventa un corollario talmente intuitivo da far pensare che non sia rimasto una mera virtualità dell'insegnamento di Anassimandro. Ora noi non siamo in grado di dire se egli parlò espressamente anche della formazione e diversificazione dei vegetali, se introdusse anche la nozione di diversificazione delle specie, né se si soffermò anche sull'energia vitale intesa come motore dell'innovazione biologica, ma che egli possa aver parlato di condizioni favorevoli per la forma- 
zione spontanea di molti tipi di esseri viventi è, per quanto io posso giudicare, del tutto verosimile, tanto più che persino Talete potrebbe aver lanciato idee che andavano nella stessa direzione.

Uno stupefacente corollario segue da quanto sopra congetturato: si direbbe che Anassimandro sia riuscito a rappresentarsi addirittura un ecosistema: piuttosto stabile, ma ,destinato' a venire progressivamente scompaginato dalla crescente siccità.

\section{La preistoria dell'ecosistema in cui noi ci troviamo a vivere. Asse spaziale e asse temporale}

Sapere che lo sguardo penetrante di Anassimandro seppe spingersi così indietro nel tempo è emozionante, ma sappiamo che in realtà egli si spinse ancora più indietro, molto più indietro. Infatti le fonti ci parlano anche della formazione dei corpi celesti per distacco da una sorta di proto-terra o proto-universo ancora indifferenziato. In particolare Eusebio di Cesarea trova il modo di riferire che, secondo Anassimandro, i corpi celesti si sono formati per distacco dalla proto-terra, che erano già allora ignei e si staccarono allo stesso modo in cui si distaccano le cortecce di alcuni grandi alberi (hōs tōi dendrōi phloion: $12 \mathrm{~A} 10 \mathrm{DK}=\mathrm{Ar} 101 \mathrm{~W}$.). Attorno alla proto-terra ci sarebbe stata dunque un cerchio (kyklos) igneo che si è frammentato e distaccato, dando luogo a sole, luna e stelle. Analogamente Ippolito (12A11.4 = Ar 75 W.) riferisce che gli astri si sono formati a partire da un cerchio di fuoco. Per la verità ambedue menzionano anche l'aria, che avrebbe circondato questo proto-universo, mentre Ippolito parla anche di un fuoco che è nel cosmo (nel tutto? nell'insieme originario?). Questi rimangono dettagli.

Invece l'idea che il proto-universo, che in origine probabilmente ruotava su se stesso come mosso da un vortice, abbia finito per dividersi in un nucleo centrale rimasto relativamente stabile e compatto, e una fascia esterna che si è distaccata e frammentata, dando poi luogo agli astri, è un dettaglio di ben altro spessore, perché il suo raccordo con altri insegnamenti è molto intuitivo. Anche se la penuria di dati specifici impone molta prudenza, non è fantasticheria postulare che, secondo Anassimandro, questa terra originaria, quando era ancora allo stato di grande ammasso pastoso e morbido, e ancora caldo, per effetto della rotazione possa essersi progressivamente schiacciata e dilatata senza perdere il profilo circolare, con la parte ignea, più mobile, che è progressivamente scivolata verso la sua periferia per poi addirittura distaccarsi (per effetto di una spinta che noi chiameremmo centrifuga). A quel punto, i corpi celesti hanno continuato - e continuano - a girare mentre si può capire che il corpo centrale abbia gradualmente perduto la sua spinta rotatoria fino a fermarsi proprio come le comuni trombe d'aria gradualmente si esauriscono e il materiale mantenuto in sospensione si deposita, favorendo il processo di solidificazione di alcune sue parti. Sussiste inoltre la possibilità che Anassimandro abbia istituito un raccordo tra la congettura sul distacco delle ,cortecce' ignee e l'attribuzione di una forma cilindrica alla terra in quanto il distacco ha interessato le propaggini più estreme di questo corpo rotondo ma schiacciato e in via di solidificazione. 
Ho così delineato una ricostruzione fortemente congetturale del probabile andamento delle trasformazioni avvenute in un passato remotissimo. A tale scopo ho congetturato che tra gli insegnamenti di Anassimandro figurasse anche la postulazione (1) di un vortice cosmogonico, (2) del progressivo schiacciamento del grande ammasso, (3) del progressivo concentrarsi del fuoco nella zona che noi diremmo equatoriale, (4) del successivo rallentamento del corpo centrale che finisce per fermarsi, (5) del nesso causale tra distacco delle, cortecce' ignee e forma attuale (cilindrica) della terra. Non siamo in grado di affermare che egli abbia svolto anche questi punti (e precisamente questi punti) ${ }^{38}$, ma sarebbe ben strano che egli non avesse collegato la proto-terra (in cui tre dei quattro elementi ancora erano approssimativamente mescolati insieme) alla terra ridotta a due elementi principali (terra-acqua che si sono venuti progressivamente diversificando e popolando), con residue tracce del fuoco e l'aria collocata all'esterno, ,attorno'. Molti fattori inducono a pensarlo: non soltanto l'omogeneità delle dinamiche ipotizzate per rendere conto delle varie fasi e il fatto di partire da un passato quanto mai remoto per arrivare addirittura al futuro (con i mari prosciugati e la terra semi-desertica), ma anche l'unicità del ciclo temporale complessivo, la capacità di rappresentarsi il sistema terra in prospettiva evoluzionistica (mi pare che un termine equivalente, ma non così moderno, non sia disponibile) e la comprovata capacità di pensare che la condizione attuale della terra sia una mera fase o tappa di un percorso fatto di innumerevoli trasformazioni. Ciò che si delinea è dunque una sorta di teoria unificata del passato remoto della terra - certamente la prima mai concepita da mente umana - che, attraverso trasformazioni graduali e diluite in un tempo indefinitamente lungo, arriva fino alla nostra condizione attuale.

Osservo inoltre che, per il fatto di collocare l'attuale disposizione dei grandi corpi nello spazio nella cornice di un ,percorso' lungo il quale anche altri equilibri si sono dati e si daranno, prende forma una inedita (quanto meditata, competente) saldatura tra quelli che sembrano costituire i due assi portanti del ,trattato', l'asse spaziale e l'asse temporale. Infatti Anassimandro ha insegnato che l'attuale disposizione di luoghi e corpi (nello spazio) non è data una volta per tutte, ma ha conosciuto (e forse potrà conoscere) delle trasformazioni anche profonde che, peraltro, non riguardano unicamente i corpi che ruotano attorno alla terra ma rendono conto anche di altre fondamentali trasformazioni, come ad es. il processo di popolamento dei mari e poi delle terre emerse.

Di riflesso, si delinea un'idea precisa del mondo, concepito come un'entità complessa, relativamente instabile e con molti attori, ma pur sempre regolata e, in certa misura, prevedibile nelle sue manifestazioni, un'entità che, oserei dire, sa provvedere a se stessa e nella quale, di nuovo, non c'è posto per gli dei dell'Olimpo. È interessante notare che l'autore non si abbandona a fantasticherie degne dei poeti nemmeno quando si tratta

\footnotetext{
38 Perilli (1996: 21-28), si sforza di giungere alla conclusione che Anassimandro effettivamente teorizzò il vortice originario, ma trovo preferibile riconoscere che sull'argomento mancano prove certe, anche se ci sono indizi a favore.
} 
di ideare una saldatura tra rappresentazione dei grandi spazi e rappresentazione di eventi cosmici disposti lungo un tempo lunghissimo.

\section{1. „Mettersi dal punto di vista della terra” e affermare senza sapere}

Le pagine precedenti dovrebbero aver fornito, oso presumere, non pochi argomenti per confidare che, dell'insegnamento di Anassimandro (e quindi del suo libro), le fonti lascino affiorare ben altro che un certo numero di tracce disorganiche e non particolarmente eloquenti. La rassegna condotta nelle pagine precedenti ha permesso, infatti, di individuare (a) molteplici insegnamenti, gran parte dei quali dotati di impressionante vitalità, (b) linee di raccordo ben poco aleatorie tra un'alta percentuale di tali insegnamenti, (c) significative tracce di ragionamenti che è probabilmente corretto (o addirittura doveroso) interpretare come tracce delle considerazioni offerte da Anassimandro a sostegno delle sue arditissime tesi. Specialmente quest'ultimo punto merita un supplemento di attenzione.

Un primo, organico, gruppo di sue considerazioni concernenti la logica che presiede alla concezione della terra (forma, posizione, fattori di stabilità, distanze...) è stato delineato nel corso del $\$ 2$; un ragionamento non meno intuitivo sostiene, se non erro, il nesso tra proto-pinax ed AU; ed anche nel caso del tentativo di utilizzare i casi di congiunzione della luna con alcuni pianeti per stimare le dimensioni della luna, comprendiamo senza difficoltà il ragionamento fatto da Anassimandro. Abbiamo poi una traccia particolarmente trasparente per quanto concerne la gestazione dei primi esseri umani, ma non molto diverso è il caso del processo di adattamento di alcuni animali marini alla vita fuori dall'acqua, processo che - possiamo fondatamente congetturare - secondo lui ha dato il via alla moltiplicazione delle specie animali terrestri nel presupposto che la terra si fosse precedentemente riempita di erbe e piante. Inequivocabile, peraltro, è anche il ragionamento in base al quale Anassimandro ha ipotizzato che, dopo la formazione dei corpi celesti, la terra ha offerto condizioni particolarmente favorevoli alla comparsa dei primissimi esseri viventi (ambiente caldo-umido e relativamente fluido), salvo poi a specificarsi in mari e superfici asciutte, in mari sempre più variamente popolati e, a seguire, terre sempre più variamente popolate. Trasparente nei suoi principi ispiratori si direbbe anche il tentativo di offrire una spiegazione unitaria dei fenomeni atmosferici $(\$ 7)$ a partire dall'idea che ogni nube sia, con la nostra terminologia, come un grande otre di origine animale che, quando si rompe per effetto della vicinanza (e quindi dell'attrito) con altri ,otri', facilmente dà luogo a ciò che noi chiamiamo tuoni, lampi e fulmini.

Si concederà che stiamo accedendo a un insieme di giustificazioni, spiegazioni e argomenti che sorprende per quanto è articolato, strutturato e, quel che più conta, retto da idee straordinariamente precise intorno a ciò che può valere come congettura plausibile. Ciò rende il pensiero di Anassimandro molto meno sfuggente e molto meno misterioso di quello di tanti altri intellettuali del periodo. A delinearsi, per queste vie, è infatti una compiuta teoria sul mondo (in modo del tutto particolare, sulla terra: per questo nel tito- 
lo propongo di parlare di „trattato sulla terra”) scandita in vasti apparati di congetture sulla disposizione dei macro-corpi nello spazio e sui macro-eventi distribuiti nel tempo. Il suo punto di forza è l'alto grado di inconfondibilità delle singole informazioni, una caratteristica che ha ben protetto le varie tessere dalla progressiva perdita di identità e che non manca di tradursi in preziosa risorsa per noi che tentiamo di ricomporre il mosaico. Parliamo infatti di congetture molte delle quali sono talmente impensate e talmente lontane dal senso comune (la singola notizia, es. quella concernente la forma cilindrica della terra, è protetta da livelli davvero altissimi di stabilità semantica: protetta, direi, quanto l'idea di centauro o di Biancaneve) che, oltre a essere molto ben protette dal rischio di stravolgimento, forniscono più di una indicazione su come erano raccordate le une alle altre.

Si tratta, peraltro, di un sapere di carattere intrinsecamente composito, nel senso che le varie teorie non si deducono l'una dall'altra. Infatti, anche se si tratta di teorie dello stesso genere, congruenti e affini dal punto di vista tipologico, accade ogni volta che la teoria $\mathrm{X}$ risulti fondarsi su considerazioni estranee alle particolari ragioni su cui si fonda la teoria $\mathrm{Y}^{39}$.

Nondimeno, Anassimandro mostra di aver individuato una sorta di binario molto caratterizzato, all'interno del quale collocare le sue molteplici congetture. Alla domanda „che pensare di ciò che noi non vediamo?” (es.: come è possibile che sole e luna passino sotto la terra? come fa la terra a non cadere?) e alla domanda „che cosa può essere accaduto?” (es. come si è potuta popolare anche la terraferma che, non essendo sufficientemente umida, dovrebbe favorire ben poco la generazione spontanea di animali? da dove viene l'acqua piovana? etc.) egli ha ricercato delle risposte semplici e di tipo - diremmo noi oggi - naturalistico, nel senso che le dinamiche ipotizzate non sono antropomorfiche, non sono stravaganti o miracolistiche, non chiamano in causa le divinità olimpiche né alcun altro ,attore' esterno antropomorfico (es. una divinità che si infuria ma non è impossibile placare). Più semplicemente, queste cause vengono ricercate in ciò che può essere rilevante per la terra stessa o per gli esseri viventi che in essa si formano, per poi venire opportunamente collocate in lungo un arco temporale di proporzioni mai immaginate prima. Gli astri, per esempio, non possono che essere derivati dalla terra per distacco di grandi masse infuocate e, se gli astri ancora ruotano, ciò vuol dire che moltissimo tempo fa ruotava tutto il sistema; ma se a ruotare fu un corpo di proporzioni grandissime come la terra, è comprensibile che un corpo ruotante così grande generasse, nei punti più lontani (noi diremmo: nella zona equatoriale) una forza centrifuga (come diremmo noi) così potente da comportare il distacco di alcuni grossi ,pezzi', distacco che ebbe dunque luogo per ragioni (diremmo noi) meramente meccaniche.

Pertanto è corretto affermare che, secondo Anassimandro, alla terra non accadono cose strane, le trasformazioni sono tutt'altro che repentine e non sono particolarmente misteriose: la terra (ha conosciuto e) conosce solo trasformazioni compatibili con ciò che

\footnotetext{
39 Su questo punto v. anche Rossetti (2004: spec. 110-139).
} 
è in grado di fare e con ciò che può ragionevolmente accaderle (ad es. senza consapevolezza degli accadimenti, senza provare emozioni, etc.). In effetti le trasformazioni ipotizzate da Anassimandro, anche quando sconvolgono gli equilibri (come nel caso delle ,cortecce' infuocate che si staccano dalla proto-terra), possono ben dirsi rette dalla medesima idea di compatibilità con le caratteristiche di base della terra e dalla medesima regolarità complessiva degli accadimenti che la concernono. Le sue congetture delineano insomma una sorta di binario fondato sull'idea che si arriva a capire qualcosa se si fa un serio sforzo per mettersi, dal punto di vista' della terra. Ciò accade e, con nostro stupore, si delinea un criterio straordinariamente appropriato per discernere tra ciò che è aspecifico, arbitrario, non-ipotizzabile (viene subito in mente il supposto ruolo di Zeus nel provocare tuoni e fulmini, ma non dimentichiamo l'idea che la terra debba necessariamente avere un non meglio identificato sostegno fisico) e ciò che si può considerare pertinente e plausibile da quel punto di vista. Il suo sapere dà prova di essere, disciplinato', anzi di istituire una ,disciplina', ossia un tipo di sapere selettivo e molto caratterizzato anche dal punto di vista della gamma di eventi o dinamiche o spiegazioni che si possono considerare proponibili ${ }^{40}$.

Che Anassimandro abbia provato - e abbia sperato di riuscire - a sollevare il velo su argomenti creduti del tutto inaccessibili è evidente. In effetti, se consideriamo l'enormità del ,viaggio' mentale da lui compiuto, possiamo ben capire che quest'uomo deve aver creduto - e vissuto l'emozione - di raggiungere risultati quasi sovrumani: per esempio, non solo rappresentare la terra (con il pinax) ma perfino, osservare' il mega-cilindro dall'esterno e addirittura ,misurarne' lo spessore; non solo dire come la terra è, ma arrivare addirittura a dire come ha fatto a diventare quella che è e come diverrà in un futuro ancora lontano... Quello fu davvero un trovarsi ad oltrepassare dei limiti creduti insuperabili per gli umani!

Eppure egli ha saputo anche darsi dei limiti superiori (ed è un altro pregio raro). Le nubi, ad es., vengono trattate come grandi otri (di pelle animale conciata), ma nulla si dice sul motivo per cui le nubi si comportano in modo analogo agli otri che un tempo erano di uso corrente; si dice che i primi pesci nacquero nel caldo-umido, ma non si prova a dire come e perché il caldo-umido comporta che nascano degli organismi viventi, semoventi, etc.; si dice che, per dare tempo ai proto-esseri-umani di crescere a sufficienza, così da non rischiare la morte, deve essere stato necessario l'intervento dei pesci (dei cetacei?), ma non ci si chiede come mai a questa funzione si sia dedicata, e con successo, una determinata specie di animali marini e non altre, tanto meno come mai l'orologio biologico dell'espulsione del feto sia stato fissato a circa 12-15 anni (etc., cf. nota 36). Il sapere di Anassimandro mostra dunque di aver individuato un ideale di conoscenza e un campo di azione assai ben delimitati, governati cioè da un ulteriore binario che serve a restringere tanto la gamma delle domande ammesse quanto la banda di oscillazione delle spiegazioni congetturali che si possono giudicare proponibili.

\footnotetext{
40 Ciò di cui la letteratura pozione non ha nemmeno provato a rendere conto non è solo il raccordo tra i vari insegnamenti, ma anche e soprattutto la disciplina intellettuale che da essi traspare.
} 
Congruente si direbbe anche l'attitudine a individuare un livello medio di attendibilità ed a non chiedere di più, nel senso che il suo sapere non punta alla certezza o all'accertamento (e tanto meno alla prova incontrovertibile) ${ }^{41}$, ma a un tipo di sapere difficile da definire, che si appaga della possibilità di farsi un'idea, sia pure approssimativa, del contesto (i territori molto lontani da Mileto, la superficie terrestre, la terra come corpo nello spazio, l'insieme dei corpi che occupano lo spazio, la vicenda evolutiva della terra e le fasi del suo popolamento, l'insieme dei fenomeni atmosferici...). Il punto di equilibrio da lui raggiunto è davvero singolare. Anche perché implica una certa spavalderia nell'affermare senza sapere. Quando ha detto che la terra è a forma di cilindro, che l'altezza del cilindro dovrebbe essere pari a un terzo del suo diametro, che il sole dista 27 volte etc. Anassimandro ha evidentemente azzardato delle valutazioni ben sapendo che esse erano del tutto inaccessibili. Anche quando ha detto che in un passato remotissimo, mentre la terra era ancora agitata da un vortice, alcune parti si staccarono e diedero luogo ai corpi celesti; anche quando ha osato sostenere che i primi animali si formarono nel caldo-umido e popolarono anzitutto i mari, dopodiché alcuni di loro riuscirono a vivere anche fuori dall'acqua e ben presto persero le scaglie perché disseccate dal sole (e in molti altri casi) egli ha ogni volta azzardato delle valutazioni del tutto inverificabili. Ma quando questa stessa persona ha disegnato, ad es., la Sicilia e l'ha collocata in un punto particolare del 'mondo' sulla base di informazioni che non poteva propriamente controllare e delle quali si è fidato, non ha forse accreditato ancora una volta, in maniera ragionevole, interi gruppi di affermazioni propriamente inverificabili, dunque senza propriamente sapere né preoccuparsi di verificare di persona?

\section{Verso una conclusione: dove collocare la nozione di apeiron e il fr. 1 ?}

L'imponenza delle informazioni che sono state passate in rassegna impone di ritornare sulla consueta emarginazione di questo immenso sapere a favore della nozione di apeiron e del fr. 1 , tante volte additati come nucleo centrale e pietra miliare dell' insegnamento del maestro. Chi fu veramente Anassimandro?

Aristotele ha concentrato la sua attenzione sull'apeiron molto più che su altri temi. Da Aristotele a tutto il secolo XX - ma anche Dúhrsen 2013 giudica appropriato dedicare a apeiron e fr. 1 ben 24 pagine delle 38 che egli dedica alle dottrine di Anassimandro è stato costante l'uso di ravvisare l'insegnamento primario di Anassimandro nella trattazione su apeiron e nel fr. $1 \mathrm{DK}$, solo marginalmente nella trattazione di altri temi ${ }^{42}$. Nondimeno, tra il sapere passato in rassegna nei paragrafi precedenti - un più che articolato

41 A costituire una novità per l'epoca fu, però, proprio la certezza fondata sul ragionamento anziché sulla constatazione (come nel caso di molte tessere dell'insegnamento di Talete), non certo il sapere un po' approssimativo di cui stiamo trattando. - Vorrei ribadire che, nondimeno, la prima rappresentazione dell'area mediterranea sembra essere stata di gran pregio anche sotto il profilo dell'attendibilità delle informazioni fornite.

${ }^{42}$ Da questo schema si seppe lodevolmente allontanare, rara avis, il Ferber in un suo breve articolo del 1986. 
sapere sulla terra - e qualche considerazione dedicata alla nozione di apeiron (particolarmente refrattaria alle precisazioni) sussiste una sproporzione addirittura spettacolare, anzitutto dal punto di vista dell'autore, che dovette investire immensamente di più nel costruire il suo sapere sui grandi spazi e i lunghi tempi della terra e dovette essere gratificato immensamente di più da queste indagini e da questi insegnamenti che non dai temi usualmente prediletti da chi guarda ad Anassimandro con gli occhiali della Metafisica e della Fisica di Aristotele (ed eventualmente delle Holzwege di Heidegger). Tra questi due blocchi non c'è proporzione. Pertanto la proporzione non può che essere rovesciata: l'occupazione centrale di Anassimandro (la sua ossessione conoscitiva, il suo principale vanto) non fu certamente l'apeiron; l'apeiron non ha certo costituito «das Grundwort Anaximanders» (Riedel 1987)! Altrimenti dovremmo dire che il secondo maestro di Mileto si è sentito mobilitato e gratificato anzitutto dalle elucubrazioni sull'apeiron (e sull'oscuro fr. 1), salvo poi a occuparsi marginalmente anche di una serie infinita di altre elaborazioni concettuali ${ }^{43}$, eventualità che, non posso negarlo, giudico semplicemente inimmaginabile. Non posso non affermare, pertanto, che l'adozione selettiva di lenti aristoteliche e heideggeriane ha comportato una spettacolare distorsione interpretativa che si dimostra, oltretutto, ancora ignara della lezione di Harold Cherniss (Aristotle's Criticism of Presocratic Philosophy, 1935).

Considerazioni imbarazzanti, ma dovute, come quelle che precedono comportano il delinearsi di un quesito inedito: dove, collocare' la sub-trattazione sull'apeiron? Si direbbe che l'apeiron possa essere stato assimilato all'immensità dello spazio vuoto nel cui ambito la terra e i corpi celesti stanno e interagiscono, e così pure all'anno zero della storia, ossia alla fase in cui sarebbe stato assolutamente prematuro parlare di terra (naturalmente possiamo anche provare a rappresentarci le due funzioni in maniera unitaria). In tal caso Anassimandro avrebbe ravvisato nell'apeiron una condizione originaria e un contesto spazio-temporale oltremodo remoto nel quale collocare la terra e insieme anche una sorta di materia prima. Ma si potrebbe dire qualcosa di più sull'argomento? Ne dubito. Quanto riferiscono Aristotele e Teofrasto su questo argomento «è così striminzito (meager) e così legato al loro schema concettuale che un esame delle evidenze solleverà

43 Tra queste, se posso nuovamente riepilogare: (a) l'invenzione e realizzazione del mappamondo con rappresentazione diagrammatica della linea costiera, (b) l'opinione che, costeggiando, deve essere possibile andare dalle Colonne d'Ercole alle Colonne d'Ercole passando per il margine orientale del Mar Nero, (c) l'idea che il sole debba continuare il suo viaggio notturno con il medesimo percorso circolare e dunque essere in grado di passare sotto la terra, (d) la ricerca e l'individuazione di un valido motivo per pensare che sia logico per la terra non precipitare nel vuoto, (e) l'idea che la terra debba costituire una immensa superficie pressoché pianeggiante, terminare con un invalicabile strapiombo e, sotto, dar luogo ad un'altra superficie anch'essa grosso modo pianeggiante e grosso modo parallela, (f) l'idea che, per chi dovesse vivere là sotto, probabilmente non ci sarà alcun bisogno di inchiodarsi al suolo per non allontanarsene definitivamente, $(\mathrm{g})$ l'idea di diametro terrestre, l'impiego di questa grandezza quale prima Astronomic Unit della storia e la connessa ipotesi che il cilindro terrestre abbia uno spessore pari ad un terzo di AU, (h) la formulazione di domande su come avranno fatto i primi uomini a sopravvivere malgrado, nei primi 12-15 anni di vita, essi siano notoriamente incapaci di provvedere a se stessi, (j) l'elaborazione di congetture su cosa può essere accaduto a quegli animali marini che, per primi, si sforzarono di sopravvivere fuori dall'acqua, $(\mathrm{k})$ l'elaborazione di congetture sull'eventualità che un tempo la terra sia stata agitata da un vortice e (i) che i corpi celesti si siano staccati dalla terra - e inoltre, come sappiamo, molto altro. 
più problemi di quanti ne possa risolvere» (Kahn 1960: 231). Indubbiamente Anassimandro ha trattato anche questo argomento, ma se da un lato non ci sono le condizioni minime per poter stabilire come precisamente la nozione di apeiron si coordinasse con quel suo sapere-sulla-terra che abbiamo appena terminato di passare in rassegna, dall'altro non possiamo non presumere che si trattasse di una tessera secondaria.

Considerazioni analoghe si applicano al fr. 1 DK. Il molto che sappiamo sul conto del pensiero di Anassimandro non ci aiuta in alcun modo a individuare un contesto appropriato per quanto viene lì proclamato. In quel frammento salta agli occhi, del resto, anche la totale assenza di contesto per la metafora giuridica, che svolge un ruolo chiave nella frase. Si può certo ipotizzare che Anassimandro, impressionato dai Nomoi di Solone in prosa, abbia voluto echeggiare il linguaggio del diritto usato dall'ateniese, ma siamo in grado di capire che uso provò a farne, e per dirci che cosa? Lungi dal costituire una risorsa di pregio per la comprensione del mondo mentale di Anassimandro, il fr. 1 rimane, pace Simplicio, una tessera isolata e una unità testuale piuttosto misteriosa (anzi, tenacemente misteriosa) su cui sarebbe azzardato, se non imprudente, speculare. Tanto più che, come ha segnalato proprio il Dúhrsen (2013: 307), l'idea di ciclicità suggerita dal fr. 1 presenta un insanabile contrasto con il processo lineare che viene delineato per la ,storia' della terra.

\section{Il libro di Anassimandro}

Il sapere di Anassimandro fu consegnato a un libro. Egli scrisse dunque un libro in cui trovava il modo di rendere conto delle sue idee intorno alla collocazione della terra in quello che noi chiamiamo spazio, e alle origini della terra a partire da un qualche indistinto originario, di non poche tappe di questa proto-storia della terra, delle verosimili misure del cosmo e di altro ancora. Del resto non è escluso che nel suo ,libro', verosimilmente scritto su cuoio conciato (cf. Hdt. V 58.3), Anassimandro possa aver inserito anche un secondo esemplare (o, se si preferisce, una copia) del proto-pinax e qualche specifica parola di commento. Vorremmo saperlo, ma è decisamente troppo tardi per poterlo appurare.

Vorremmo sapere anche molte altre cose, perché la natura del suo sapere ci invita a pensare che egli abbia saputo far posto alle varie sub-trattazioni con un certo ordine, se non altro nel disporre le tappe della proto-storia della terra secondo un criterio cronologico (es.: „dapprima avvenne il distacco delle cortecce di fuoco, poi la distinzione tra terra e mare e l'avvio di un lento ma inesorabile prosciugamento”; „prima ebbe luogo la formazione dei pesci e delle piante, poi fu la volta degli animali terrestri, infine dei primi uomini neopuberi”). Non poter accertare nemmeno questo è davvero una grande perdita, ma il fatto che il sapere di Anassimandro si disponga così chiaramente lungo alcune grandi direttrici o aree nitidamente identificabili (di nuovo: il pinax, i grandi spazi con la terra stabile nel vuoto, la meteorologia, il passato remoto della terra, il suo futuro remoto) e di poter ammirare la disciplina intellettuale che egli seppe imporsi, ci parla 
inequivocabilmente di un ordine mentale di cui avrà risentito anche l'organizzazione di un ,libro' necessariamente costituito da molteplici sub-trattazioni.

A maggior ragione siamo autorizzati a presumere che Anassimandro abbia dato un contributo decisivo all' invenzione del trattato inteso come deposito (un deposito non troppo disordinato) di conoscenze, insomma all'invenzione di un tipo molto particolare di ,libro', il Peri physeos. Questo tipo di scritti è riconoscibile non solo per le tematiche più spesso ricorrenti, per il tipo di sapere che viene proposto e per gli indizi di continuità di una ricerca condotta da molti intellettuali presocratici, nonché per il titolo ${ }^{44}$, ma anche e soprattutto per il fatto di aver dato vita, senza poter contare su alcun precedente, a un modello credibile di libro-archivio, di libro di testo, di libro-summa di un certo tipo di conoscenze mediamente dotate di ratio. Se poi si considera la funzione didascalica dei poemi omerici, è inevitabile concludere che il libro di Anassimandro - il primo della serie - dovette costituire, rispetto a quel tipo di scritti, un'alternativa più che credibile, tanto è vero che ha dato luogo a tanti altri Peri Physeos analoghi, un po' come il protopinax ha dato luogo a tanti altri pinakes. Spiace solo dover constatare che le sue idee si rivelarono troppo avanzate per poter essere comprese e prese in seria considerazione dai contemporanei, per cui non ci volle meno di un Parmenide per riscoprirne almeno alcune e, partendo da lì, ricominciare con nuove congetture non troppo dissimili dalle sue nell'impostazione.

Concludo: di quante cose Anassimandro merita di essere considerato „padre”? Della cartografia, della geografia, della AU, del principio di inerzia, dell'astronomia, della storia geologica e dell'evoluzionismo, della teoria degli ecosistemi, del ciclo delle acque, della meteorologia, del trattato, del libro di testo... Siamo sicuri che l'elenco sia completo?

\section{Infine: Anassimandro e Talete}

Talete costituisce il termine di paragone più diretto ed è possibile che sia stato l'unico interlocutore, il primo critico e il più immediato punto di riferimento di Anassimandro. Anche Talete è stato vittima di rappresentazioni straordinariamente riduttive, che sono state segnate, in particolare, da una incongrua dipendenza da Aristotele, come se il tema dell'acqua fosse stata la scoperta di cui egli poté andare maggiormente fiero, mentre siamo in grado di escludere del tutto una simile eventualità ${ }^{45}$. In realtà Talete si fece un nome soprattutto grazie a molte misurazioni ,impossibili' ${ }^{26}$, e ciò permette di capire la differenza con l'allievo: Anassimandro ha polarizzato la sua attenzione su elementi

\footnotetext{
${ }^{44}$ In proposito v. anche Rossetti (2006).

45 Mi basti osservare che Talete deve essere stato, anzitutto, un maestro in misurazioni e in particolare nell'acquisizione di dati quantitativi tutt'altro che approssimativi in una sorprendente varietà di campi molto diversi tra loro (es. misurazione di distanze, misurazione di durate).

${ }^{46}$ Congetture su alcune di queste misurazioni sono state proposte in Rossetti (2013a).
} 
sistemici (tutta la terra, tutto il mondo, la storia dell'intero universo...) ed ha cercato di capire la logica di vasti insiemi di fenomeni e processi accettando, come contropartita, di fare affermazioni molto meno controllabili, di non poter propriamente provare le sue tesi e, solo in una sparuta minoranza di casi ${ }^{47}$, di poter montare apparati per il calcolo alla maniera di Talete. Invece Talete aveva coltivato l'aspirazione ad effettuare accertamenti quantitativi - spesso di pregio - su questioni particolari e solo su questioni particolari, anche se significative. Per queste ragioni, il suo sapere ebbe il dono della verificabilità e della piena comprensibilità (nel senso che molti, con il necessario addestramento e un po' di applicazione, potevano arrivare a comprendere nitidamente come e perché si riesce a stabilire la data di solstizi ed equinozi così come a misurare l'altezza di una piramide o l'ampiezza angolare del sole). Inoltre i suoi accertamenti, per il fatto di aprire uno spiraglio sul conto di svariati segreti molto ben protetti (dall'ampiezza angolare del sole all'irregolare durata delle stagioni, dalla natura delle eclissi alla misurazione indiretta di altezze inaccessibili), finirono per dare il via ad una sorta di tremenda hybris conoscitiva, hybris che contagiò profondamente anche il discepolo.

Malgrado questo punto di contatto costitutivo, ciò che sembra predominare è la discontinuità: discontinuità fra la passione per le misurazioni del maestro e le immense energie investite dal discepolo nel tentativo di rappresentarsi e capire aspetti costitutivi del macro-contesto spaziale e temporale in cui ci troviamo a vivere (in particolare: discontinuità fra la passione per gli accertamenti quantitativi del maestro e la consapevole rinuncia del discepolo ad appurare, tanto da osare accontentarsi di misure solo indicative) e discontinuità fra due modi, ognuno molto caratterizzato e coerente, di concepire i fini e i mezzi dell' indagine. L'allievo è stato non meno creativo del maestro nel delineare un suo progetto di ricerca e una sua idea di sapere che si è rivelata, possiamo ben dirlo, oltremodo innovativa e feconda.

Dunque due indagatori infaticabili, due proto-scienziati ma non due filosofi? Qui mi basti dire che il loro sapere ebbe un formidabile potenziale filosofico (anche se per meriti ai quali, pace Aristotele e seguaci, poco dovrebbero aver contribuito le riflessioni sull'archē e/o sull'apeiron) in quanto, come si usa dire, essi hanno spalancato orizzonti nuovi, istituito possibilità del tutto nuove per la mente, esaltato la possibilità di oltrepassare i limiti correnti nel venire a sapere e nel riuscire a capire; inoltre hanno avuto bisogno di spiegare (o tentar di spiegare) ciò che avevano congetturato o calcolato, e sono preziose le tracce di questi loro ragionamenti giudiziosi - ad es. l'analogia che Talete seppe istituire tra la 'pietra' di Magnesia e l'ambra strofinata, accomunati dalla stessa capacità di provocare effetti visibili (l'attrazione, l'induzione a spostarsi, l'induzione a rimanere attaccati...), analogia in forza della quale egli concluse che gli oggetti inanimati (apsuchoi)

\footnotetext{
${ }^{47}$ La data del tramonto delle pleiadi e lo gnomone (cf. $\$ 6 a$ e $\left.6 c\right)$.
} 
non dovrebbero essere propriamente e totalmente inanimati come il nome lascerebbe intendere (Diog. Laert. I 24).

Presumo dunque che il loro apporto alla filosofia sia ravvisabile anzitutto nell'originalità e fecondità del loro modo di essere ricercatori e maestri, quindi per esempio nella loro capacità di impegnarsi a fondo in molteplici tentativi di accertare e/o capire qualcosa, nella loro capacità di darsi una disciplina intellettuale per poi formulare quesiti e impostare percorsi in grado di condurre a risposte di pregio: qualità che poterono entusiasmare, tanto più che essi diedero prova di una creatività inaudita, se è vero che, non a caso, molti dei loro insegnamenti sono diventati patrimonio dell'umanità. Direi, invero, che sia stata soprattutto la loro condizione di ricercatori a fare scuola. In questo senso, a dovere moltissimo ai due primi maestri di Mileto non è solo la filosofia, né è solo la scienza: è l'umanità ${ }^{8}$.

${ }^{48}$ Ho potuto utilmente discutere alcuni punti di questa indagine con Guido Calenda, Dirk Couprie e Carlo Rovelli e, sotto il profilo dell'epistemologia, con Luigi Cimmino e Marco Bastianelli. 


\section{BIBLIOGRAFIA}

Barnes, J., 1979, The Presocratic Philosophers, London-Henley-Boston.

Betegh, G., 2001, «Le problème des représentations visuelles dans la cosmologie présocratique: pour une histoire de la modélisation», in A. Laks-C. Loguet (eds.), Qu'est-ce que la philosophie présocratique? / What is Presocratic Philosophy?, Villeneuve d'Ascq, pp. 381-415.

Cerri, G., 2007, «L'oceano di Omero: un'ipotesi nuova sul percorso di Ulisse», in E. Greco-M. Lombardo (eds.), Atene e l'Occidente. I grandi temi. Atti ... 2006, Atene, pp. 13-51.

Cleve, F.M., 1965, The Giants of Pre-Sophistic Greek Philosophy, The Hague.

Corre, J.-F., 2010, «Le gnomon d'Anaximandre», Revue de Philosophie Ancienne 28.2, pp. 3-31.

Corre, J.-F., 2013, «Proportions du ciel d'Anaximandre», Phronesis 58, pp. 1-16.

Couprie, D.L., 2011, Heaven and Earth in Ancient Greek Cosmology, From Thales to Heraclides Ponticus, New York-Dordrecht-Heidelberg-London.

Coxon, A.H., 2009, The Fragments of Parmenides, Edited with New Translations by R. McKirahan, Las Vegas [prima ed.: Assen 1986].

DúHrsen, N.C., 2013, «Anaximander», in H. Flashar-D. Bremer-G. Rechenauer (eds.), Frühgriechische Philosophie (Die Philosophie der Antike 1), Basel, pp. 263-320.

Ferber, R., 1986, «Der Ursprung der Wissenschaft bei Anaximander von Milet», Theologie und Philosophie 61 , pp. 551-561.

Forbiger, A., 1842, Handbuch der alten Geographie, Band I, Leipzig.

GEHRKE, H.-J., 1998, «Die Geburt der Erdkunde aus dem Geist der Geometrie. Überlegungen zur Entstehung und zur Frühgeschichte der wissenschaftlichen Geographie bei den Griechen», in W. Kullmann-J. AlthoffM. Asper (eds.), Gattungen wissenschafticher Literatur in der Antike, Tübingen, pp. 165-192.

Hahn, R., 2001, Anaximander and the Architects. The Contribution of Egyptian and Greek Architectural Technologies on the Origins of Greek Philosophy, Albany.

HAHN, R. 2010, Archaeology and the Origins of Philosophy, Albany.

HARLEY, J.B., Woodward, D., The History of Cartography, volume one, Cartography in Prehistoric, Ancient and Medieval Europe and the Mediterranean, Chicago \& London.

HeILeN, S., 2000, «Die Anfänge der wissenschaftlichen Geographie», in G. Wöhrle (ed.), Geschichte der Mathematik und der Naturwissenschaften, II, Stuttgart, pp. 35-54.

KaHN, CH. H., 1960, Anaximander and the Origins of Greek Philosophy, New York.

Kirk, G.S., Raven, J.E., SCHOFIELD, M., 1983, The Presocratic Philosophers, Cambridge [KRS].

NAdDAF, G., 2001, «Anaximander's Measurements Revisited», in A. Preus (ed.), Before Plato. Essays in Ancient Greek Philosophy, VI, Albany, pp. 5-21.

Perilli, L., 1966, La teoria del vortice nel pensiero antico. Dalle origini a Lucrezio, Ospedaletto PI.

Popper, K. R., 1958, «Back to the Presocratics», Proceedings of the Aristotelian Society 59, pp. 1-24.

RIEDEL, M., 1987, «Arche und Apeiron. Über das Grundwort Anaximanders», Archiv für Geschichte der Philosophie 69, pp. 1-17.

Rovelli, C., 2011, Che cos'è la scienza. La rivoluzione di Anassimandro, Milano [ed. francese: Paris 2009].

Rossetti, L., 2004, «Empedocle scienziato», in L. Rossetti-C. Santaniello (eds.), Studi sul pensiero e la lingua di Empedocle, Bari, pp. 95-198.

RossetTi, L., 2006, «Caratteristiche tipologiche dei trattati Peri physeos», Nova Tellus 24.2, 2006, pp. 111-146. 
Rossetti, L., 2013a, «Cominciare a misurare il cosmo. La precisione di cui fu capace Talete e il sole „largo quanto un piede umano" (Eraclito)», B@belonline 13, pp. 35-52.

Rossettr, L., 2013b, «Anassimene vs. Anassimandro», in A. Gostoli-G. Velardi (eds.), Mythologein. Mito e forme di discorso nel mondo antico. Studi in onore di Giovanni Cerri, Roma [in prep.].

RossetTi, L., 2013c, « L'ideazione del pinax, 'mediale Innovation' di Anassimandro», in D.F. Leão-G. CornelliM.C. Peixoto (eds.), Dos homens e suas ideias: estudos sobre as Vidas de Diógenes Laércio, Coimbra-São Paulo, pp. 89-100.

WöHrLe, G., 2009, Die Milesier: Thales, Berlin.

WöHRLE, G., 2011, Die Milesier: Anaximander und Anaximenes, Berlin.

LIVIO ROSSETTI

/Perugia/

\section{Anaximander's Treatise on the Earth}

The present paper argues that the teachings of Anaximander are much better knowable than they actually appear, since a number of his teachings have the privilege of being almost transparent in their predicative content as well as in their logic. As a matter of fact, one can quite easily come to understand the train of thought which lies behind Anaximander's most momentous conjectures. Thus, a largely unexpected Anaximander comes to light despite the availability of the majority of the relevant sources since 1903. Two main areas appear to be particularly prominent: on the one hand, the complex body of various conjectures and doctrines that helps to understand the system of spatial relationships from Miletus to the stars and, on the other hand, the equally complex body of conjectures and doctrines whose primarily concern is the macro-story of the Earth from its most remote past to its predictable future. The merits of Anaximander as an earth-researcher are much greater than one could actually imagine. It is suggested here that what philosophy owes to him in particular lies in his quest for knowledge, his method, his cognitive hybris, and his intellectual discipline, rather than individual doctrines. A comparison with Thales follows in the last paragraph.

Anaximander, physics, cosmology, the Earth, cartography, Thales, Anaximenes, Homer. 